\title{
Distinct Functions of Syntaxin-1 in Neuronal Maintenance, Synaptic Vesicle Docking, and Fusion in Mouse Neurons
}

\author{
Gülçin Vardar, ${ }^{1,2}$ @Shuwen Chang, ${ }^{1,2}$ Marife Arancillo, ${ }^{2}$ ○Yuan-Ju Wu, ${ }^{2}$ Thorsten Trimbuch, ${ }^{1,2}$ \\ and $\oplus^{-C h r i s t i a n ~ R o s e n m u n d ~}{ }^{1,2}$ \\ ${ }^{1}$ Department of Neurophysiology and ${ }^{2}$ NeuroCure Cluster of Excellence, Charité Universitätsmedizin Berlin, 10117 Berlin, Germany
}

\begin{abstract}
Neurotransmitter release requires the formation of soluble $\mathrm{N}$-ethylmaleimide-sensitive factor attachment protein receptor (SNARE) complexes by SNARE proteins syntaxin-1 (Stx1), synaptosomal-associated protein 25 (SNAP-25), and synaptobrevin-2 (Syb2). In mammalian systems, loss of SNAP-25 or Syb2 severely impairs neurotransmitter release; however, complete loss of function studies for Stx1 have been elusive due to the functional redundancy between Stx1 isoforms Stx1A and Stx1B and the embryonic lethality of Stx1A/1B double knock-out (DKO) mice. Here, we studied the roles of Stx1 in neuronal maintenance and neurotransmitter release in mice with constitutive or conditional deletion of Stx1B on an Stx1A-null background. Both constitutive and postnatal loss of Stx1 severely compromised neuronal viability in vivo and in vitro, indicating an obligatory role of Stxl for maintenance of developing and mature neurons. Loss of Munc18-1, a high-affinity binding partner of Stx1, also showed severely impaired neuronal viability, but with a slower time course compared with Stx1A/1B DK0 neurons, and exogenous Stx1A or Stx1B expression significantly delayed Munc18-1-dependent lethality. In addition, loss of Stx 1 completely abolished fusion-competent vesicles and severely impaired vesicle docking, demonstrating its essential roles in neurotransmission. Putative partial SNARE complex assembly with the SNARE motif mutant Stx1A ${ }^{\mathrm{AV}}$ (A240V, V244A) was not sufficient to rescue neurotransmission despite full recovery of vesicle docking and neuronal survival. Together, these data suggest that Stxl has independent functions in neuronal maintenance and neurotransmitter release and complete SNARE complex formation is required for vesicle fusion and priming, whereas partial SNARE complex formation is sufficient for vesicle docking and neuronal maintenance.
\end{abstract}

Key words: autapse; cell culture; Munc18; SNARE; vesicle priming

Significance Statement

Syntaxin-1 (Stx1) is a component of the synaptic vesicle soluble $N$-ethylmaleimide-sensitive factor attachment protein receptor (SNARE) complex and is essential for neurotransmission. We present the first detailed loss-of-function characterization of the two Stxl isoforms in central mammalian neurons. We show that Stx1 is fundamental for maintenance of developing and mature neurons and also for vesicle docking and neurotransmission. We also demonstrate that neuronal maintenance and neurotransmitter release are regulated by Stxl through independent functions. Furthermore, we show that SNARE complex formation is required for vesicle fusion, whereas partial SNARE complex formation is sufficient for vesicle docking and neuronal maintenance. Therefore, our work provides insights into differential functions of Stxl in neuronal maintenance and neurotransmission, with the latter explored further into its functions in vesicle docking and fusion.

\section{Introduction}

Syntaxin-1 (Stx1) is a neuronal soluble $N$-ethylmaleimidesensitive factor attachment protein receptor (SNARE) protein located at the plasma membrane (t-SNARE) and involved in

Received April 21, 2016; revised May 20, 2016; accepted June 9, 2016.

Author contributions: G.V. and C.R. designed research; G.V., S.-W.C., M.A., Y.-J.W., and T.T. performed research; G.V. analyzed data; G.V. and C.R. wrote the paper.

This work was supported by the NeuroCure Cluster of Excellence Exc257 and the Medical Neuroscience PhD training program (G.V.), the European Research Council (Advanced Grant SynVGlut), and the German Research Council (Collaborative Research Grant SFB958 to C.R.). We thank Mathjis Verhage for the Munc18-1 K0 mouse line; synaptic transmission by interactions with another neuronal t-SNARE, synaptosomal-associated protein 25 (SNAP-25), and the vesicle-SNARE (v-SNARE), synaptobrevin-2 (Syb2). The

the Charité Viral Corefacility and Katja Pötschke, Bettina Brokowski, Berit Söhl-Kielczynski, Rike Dannenberg, Sabine Lenz, and Pascal Fenske for technical assistance; Eric Reynolds for comments on the manuscript; and Melissa Herman for discussions and critical feedback on the manuscript.

The authors declare no competing financial interests.

Correspondence should be addressed to Christian Rosenmund, NeuroCure Cluster of Excellence, Charité Universitätsmedizin Berlin, Charitéplatz 1, Charité-Cross-0ver, 10117 Berlin, Germany. E-mail: christian.rosenmund@charite.de.

DOI:10.1523/JNEUROSCI.1314-16.2016

Copyright $\odot 2016$ the authors $\quad 0270-6474 / 16 / 367911-14 \$ 15.00 / 0$ 
$\alpha$-helical SNARE motifs of Stx1, SNAP-25, and Syb2 form the ternary SNARE complex, which is thought to catalyze vesicle fusion by bringing vesicular and plasma membranes in close proximity (Rizo and Rosenmund, 2008; Rizo and Südhof, 2012).

Gene deletion studies of the neuronal SNARE proteins in vertebrate and invertebrate model organisms illustrate the crucial roles of the SNAREs in vesicle fusion and in the preceding preparatory steps, vesicle docking and priming. Both in Syb2 knock-out $(\mathrm{KO})$ mice and SNAP-25 KO mice, $\mathrm{Ca}^{2+}$ - evoked neurotransmitter release is abrogated, but a low level of spontaneous neurotransmitter release is sustained (Schoch et al., 2001; Washbourne et al., 2002; Delgado-Martínez et al., 2007). However, loss of the neuronal Sec1/Munc18-like protein Munc18-1, the tight binding partner of Stx1 (Hata et al., 1993), results in a remarkably more severe phenotype in mammals with a complete inhibition of neurotransmitter release accompanied by neuronal degeneration (Verhage et al., 2000), with similar results obtained in flies (Harrison et al., 1994).

The widespread neuronal degeneration in Munc18-1 KO mice was first proposed as a direct consequence of lack of synaptic input (Verhage et al., 2000). However, this proposal was challenged by the findings from a Munc13-1/2 double KO (DKO) mouse model, which is also completely synaptically silent but without any cytotoxic effects (Varoqueaux et al., 2002). Ultimately, it was proposed that the underlying mechanism for the cell-autonomous degeneration of Munc18-1 KO neurons is related to another function of Munc18-1 independent of synaptic transmission (Heeroma et al., 2004). So far, only deletion of Stx 1 in flies has manifested an equally severe phenotype among SNAREs, with a complete inhibition of neurotransmission accompanied by cell lethality (Schulze et al., 1995; Schulze and Bellen, 1996; Burgess et al., 1997). The tight interactions between Stx1 and Munc18-1 and the similarities between their loss-offunction phenotypes strongly suggest the involvement of Stx1 and Munc18-1 as a complex in neuronal maintenance.

In mammals, Stx1 has two ubiquitously expressed neuronal isoforms, Stx1A and Stx1B, which share 84\% homology (Bennett et al., 1992). Investigation of Stx1 function in neurotransmitter release has been rendered impracticable in mammals for two main reasons: mutual compensatory effects of Stx1A and Stx1B (Fujiwara et al., 2006; Gerber et al., 2008; Arancillo et al., 2013; Zhou et al., 2013; Wu et al., 2015) and embryonic lethality due to constitutive deletion of Stx1A/1B (Mishima et al., 2014). Here, we present a comprehensive study of Stxl's roles in neuronal maintenance and neurotransmitter release by bypassing these obstacles through the utilization of two Stx1A/1B DKO mouse models. The first, a constitutive deletion of Stx1A/1B, led to severe neuronal lethality in vitro and in vivo, indicating the absolute requirement for Stx1 in neuronal maintenance. By using the second mouse model, a Stx1A/1B conditional DKO (cDKO), we showed that deletion of Stx1 in mature neurons also caused severe impairments in neuronal viability, demonstrating Stx1 dependency for maintenance throughout the neuronal life span. In addition, loss of Stx1 completely inhibited vesicle fusion and priming and resulted in significant reduction in docked vesicles, showing the essential roles of Stx1 for each step of neurotransmitter release: vesicle docking, priming, and fusion. Finally, exogenous expression of a mutant isoform Stx1A ${ }^{\mathrm{AV}}$ (A240V, V244A), which forms SNARE complexes with reduced stability (Kee et al., 1995, Fergestad et al., 2001), rescued neuronal survival and vesicle docking in Stx1-deficient neurons, but could not rescue any form of vesicle fusion. We conclude that the func- tions of Stx1 in neuronal maintenance and neurotransmitter release are independent from one another, that full SNARE complex assembly is crucial for vesicle fusion and priming, and that partial SNARE complex assembly is sufficient for vesicle docking.

\section{Materials and Methods}

Animal maintenance and generation of mouse lines. All procedures for animal maintenance and experiments were in accordance with the regulations of and approved by the animal welfare committee of Charite Medical University and the Berlin state government Agency for Health and Social Services under license number T0220/09. The generation of Stx1A KO $(-/-)$, Stx1B Het $(+/-), S_{x} 1 B^{\mathrm{FL} / \mathrm{FL}}$, and Munc18-1 KO mouse lines were described previously (Verhage et al., 2000; Gerber et al., 2008; Arancillo et al., 2013; Wu et al., 2015). Stx1A KO mice were intercrossed with Stx1B Het or Stx1B ${ }^{\mathrm{FL} / \mathrm{FL}}$ to generate Stx1A/1B DKO or Stx1A/1B cDKO mouse lines, respectively.

Histology and Western blot. Mouse embryos of either sex were obtained by cesarean section of pregnant females from timed heterozygous breedings. For histological analysis of the brain tissues, whole heads of mouse embryos at embryonic day (E) 16 and E18 were immersion fixed in 4\% paraformaldehyde (PFA) in $0.1 \mathrm{M}$ sodium phosphate buffer (PB) for two nights at $4^{\circ} \mathrm{C}$. The tissues were incubated in $0.4 \mathrm{M}$ and then $0.8 \mathrm{M}$ sucrose in $0.1 \mathrm{M} \mathrm{PB}$, overlaid with Tissue-Tek, and snap-frozen in n-hexane at $-70^{\circ} \mathrm{C}$ for cryoprotection. The tissues were then cryosectioned sagittally using an HM 500 OM cryostat (MICROM International) with a thickness setting of $20 \mu \mathrm{m}$. The sections were mounted directly on gelatincoated glass slides. Every sixth section was subjected to Nissl staining with $0.2 \%$ cresyl violet acetate in $20 \mathrm{~mm}$ acetic acid solution and then examined under an Olympus SZX16 microscope with an Olympus DP70 digital camera. For Western blot, whole-brain lysates were obtained from E16 or E18 embryos depending on the experiment. The samples were dissolved in lysis buffer containing $50 \mathrm{~mm}$ Tris/ $\mathrm{HCl}, \mathrm{pH}$ 7.9, $150 \mathrm{~mm}$ $\mathrm{NaCl}, 5$ mm EDTA, 1\% Triton X-100, 0.5\% sodium deoxycholate, $1 \%$ Nonidet P-40, and 1 tablet of Complete Protease Inhibitor (Roche) for 10 $\mathrm{ml}$ of solution. Equal amounts of solubilized proteins were loaded in $8-12 \%$ SDS-PAGE and subsequently transferred to nitrocellulose membranes. The membranes were subjected to the following primary antibodies overnight at $4^{\circ} \mathrm{C}$ : mouse monoclonal anti- $\beta$-tubulin III (1:2000) as an internal control, rabbit polyclonal Munc18-1 (1:2000; both purchased from Sigma-Aldrich), mouse monoclonal anti-Stx1A (1:1000), rabbit polyclonal anti-Stx1B (1:1000), mouse monoclonal anti-SNAP-25 (1:2000), mouse monoclonal anti-Syb-2 (1:2000), rabbit polyclonal antiMunc13-1 (1:1000), mouse monoclonal anti-synaptotagmin-1 (1:1000), rabbit polyclonal anti-complexin-1/2 (1:500), and mouse monoclonal anti-Rab-5 (1:500; all purchased from Synaptic Systems). Horseradish peroxidase-conjugated goat secondary antibodies (Jackson ImmunoResearch) were applied for $1 \mathrm{~h}$ at room temperature and detected with ECL Plus Western Blotting Detection Reagents (GE Healthcare Biosciences) in Fusion FX7 image and analytics system (Vilber Lourmat). Ratiometric quantification of protein expression levels was performed using ImageJ software. Data from Stx1A KO and Stx1B Het/Stx1A KO animals were pooled together after no significant differences were observed between these genotypes.

Neuronal cultures and neuronal count. Cortical cultures were prepared from E16 mice of either sex, which were obtained by cesarean section of pregnant females from timed heterozygous breedings. Hippocampal cultures were prepared from mice of either sex at postnatal day (P) $0-2$ as described previously (Xue et al., 2007; Arancillo et al., 2013). Briefly, the neurons were seeded with densities as follows: $50 \times 10^{3} /$ well $(22 \mathrm{~mm}$ diameter) for neuronal counting, $30 \times 10^{3} /$ well $(22 \mathrm{~mm}$ diameter $)$ for immunocytochemistry on continental astrocyte feeder layers as highdensity cultures, and $3 \times 10^{3}$ neurons/well ( $35 \mathrm{~mm}$ diameter) for electrophysiology on micro-island astrocyte feeder layers. The astrocyte feeder layers were prepared 1-2 weeks before the neuronal culture preparation as described previously (Arancillo et al., 2013). The cortical cultures were transduced with lentiviruses immediately after plating at day in vitro (DIV) 0 and the hippocampal cultures were transduced at DIV 
1-7 by lentiviral infection according to the experiment. The cultures were incubated in Neurobasal-A medium supplemented with B-27 (Invitrogen), $50 \mathrm{IU} / \mathrm{ml}$ penicillin, and $50 \mu \mathrm{g} / \mathrm{ml}$ streptomycin at $37^{\circ} \mathrm{C}$ between the days of neuronal counting or until DIV 13-17 before immunocytochemical or electrophysiological analysis.

The viability of the neurons in vitro was defined as the number of surviving neurons at different time points between DIV 1 and DIV 42 according to the experiment. Phase-contrast bright-field images of 10 randomly selected fields of $1.23 \mathrm{~mm}^{2}$ per well and of 2 wells per group in each culture were acquired with a DMI4000 microscope, DFC 345 FX camera, HCX PL FLUOTAR 10× objectives, and LAS-AF software (all from Leica). The neurons between DIV 1 and DIV 7 were counted manually with ImageJ software. After DIV 7, when the nuclear localization signal (NLS)-green fluorescent protein (GFP) and/or NLS-red fluorescent protein (RFP) were at adequate levels for detection, additional fluorescent images were acquired with excitation wavelengths of 488 and $555 \mathrm{~nm}$ from the same selected fields and the neurons were counted with the 3D Objects Counter function in Fiji software. To determine the survival of the Stx1A/1B DKO and Munc18-1 KO neurons, the number of neurons in each group was normalized to the number of seeded neurons as a common reference, whereas the rescue efficiencies of lentiviral reintroduction of Stx1A, Stx1B, or Munc18-1 were calculated as the normalized ratio of the number of surviving neurons to the number of Stx $1 \mathrm{~A} \mathrm{KO}$ or wild-type (WT) neurons, respectively. To evaluate the rates of cell death of Stx1A/1B cDKO neurons expressing Cre recombinase, the number of neurons was normalized to the number of neurons in control groups at DIV 7 for the cultures infected at DIV 1. For the cultures infected at DIV 3, 5, or 7, the number of neurons within each group at DIV 7 was used as reference for normalization. The ability of WT or mutant Stx1A to rescue the neurons against cell death was examined by coinfection with viruses to express Cre recombinase and determined as the ratio of the number of neurons at DIV $24-25$ to the number of neurons at DIV 11.

Lentiviral constructs. All lentiviral constructs were based on the vector FUGW (Lois et al., 2002). The cDNA for mouse Stx1A (NM_016801), mouse Stx1B (NM_024414), or rat Munc18-1 (NM_013038) were cloned after a NLS-GFP-P2A expression cassette controlled by human synapsin- 1 promoter. The P2A sequence allows for the bicistronic expression of nuclear-targeted GFP and the corresponding cDNA (Kim et al., 2011). Empty vectors, including only an NLS-GFP-P2A or NLS-RFPP2A expression cassette, served as controls. A lentivirus construct, which induces expression of improved Cre recombinase (iCre) fused to NLSRFP-P2A under control of human synapsin-1 promoter, was used to permit excision of the LoxP flanked Stx1B DNA. The point mutations A240V and V244A were introduced into Stx1A cDNA (Stx1A ${ }^{\mathrm{AV}}$ ) with the QuikChange Site-Directed Mutagenesis Kit (Stratagene). All lentiviral particles were provided by the Viral Core Facility of the Charite Berlin and were prepared as described previously (Lois et al., 2002). Briefly, HEK293T cells were cotransfected with $10 \mu \mathrm{g}$ of shuttle vector, $5 \mu \mathrm{g}$ of helper plasmids pCMVdR8.9, and $5 \mu \mathrm{g}$ of pVSV.G with X-tremeGENE 9 DNA transfection reagent (Roche Diagnostic). Virus containing cell culture supernatant was collected after $72 \mathrm{~h}$ and filtered for purification. Aliquots were flash-frozen in liquid nitrogen and stored at $-80^{\circ} \mathrm{C}$. Viruses were titrated visually with mass-cultured WT hippocampal mouse neurons using the fluorescence reporter. For infection, $\sim 1 \times 10^{6}$ infectious virus units per 22 - or $35-\mathrm{mm}$-diameter well were added onto the cultured hippocampal or cortical mouse neurons between DIV 0 and 7 depending on the experiment.

Immunofluorescence labeling. High-density cortical cultures were fixed at DIV 2, 4, or 22, whereas high-density hippocampal cultures were fixed at DIV 12, 15, or 21 . Both cultures were fixed in 4\% PFA for $20 \mathrm{~min}$, permeabilized with $1 \times$ PBS containing $0.1 \%$ Tween 20 (PBS-T) for 20 min, and blocked with PBS-T containing $2.5 \%$ normal goat serum (NGS) and $2.5 \%$ normal donkey serum (NDS) for $1 \mathrm{~h}$. The cortical cultures fixed at DIV 2 and 4 were incubated overnight at $4^{\circ} \mathrm{C}$ with chicken polyclonal anti-MAP2 (Merck) primary antibody (1:2000) in PBS-T containing NGS and NDS. The cortical cultures fixed at DIV 22 and the hippocampal cultures were subjected to guinea pig polyclonal antiBassoon (Synaptic Systems) primary antibody (1:2500), which was used as a synaptic marker, in addition to the primary antibodies against Stx1A (1:1000) and Stx1B (1:1000) mentioned above, overnight at $4^{\circ} \mathrm{C}$. Subsequently, the cells were incubated in the following fluorescently labeled secondary antibodies (1:500) according to the experiments: Alexa Fluor 647 (A647) donkey anti-chicken IgG or A647 donkey anti-mouse IgG, rhodamine red donkey anti-guinea pig IgG (all purchased from Jackson ImmunoResearch), and Pacific blue goat anti-rabbit IgG (Invitrogen). The coverslips were mounted on glass slides with MOWIOL mounting agent (Sigma-Aldrich). Immunofluorescence labeling was visualized under an Olympus IX81 epifluorescent microscope with $60 \times$ optical magnification, MicroMax:1300YHS camera (Princeton Instruments) and MetaMorph software (Molecular Devices) using $Z$-stacks. The images were analyzed offline with ImageJ as described previously (Arancillo et al., 2013). Briefly, the $Z$-stacks were overlapped with maximum intensity and the background was subtracted with a rolling ball of a radius of 30 pixels. Two to four regions exhibiting only neuronal extensions were cropped per image and analyzed as independent measurements. Bassoon-positive spots in these crops were determined using the threshold function with MaxEntropy and the created areas were saved as ROIs. The intensities of Bassoon, Stx1A, and Stx1B signals were measured using the corresponding ROIs. The average ratio of the Stx1A and Stx1B signals to the Bassoon signal was calculated for each crop and the ratios were normalized to the control group.

Electrophysiology. Whole-cell patch-clamp recordings were performed on autaptic hippocampal neurons at DIV 13-17 at room temperature with a Multiclamp 700B amplifier controlled by Clampex 9.2 software (both from Molecular Devices). Data were digitally sampled at $10 \mathrm{kHz}$ and low-pass filtered at $3 \mathrm{kHz}$ with an Axon Digidata 1322A digitizer (Molecular Devices). The standard extracellular solution was applied with a fast perfusion system $(1-2 \mathrm{ml} / \mathrm{min})$ and contained the following (in mM): $140 \mathrm{NaCl}, 2.4 \mathrm{KCl}, 10 \mathrm{HEPES}, 10$ glucose, $2 \mathrm{CaCl}_{2}$, and $4 \mathrm{MgCl}_{2}$ ( $\sim 300$ mOsm; $\mathrm{pH} 7.4)$. Borosilicate glass patch pipettes were pulled with a multistep puller, yielding a final tip resistance of $2-5 \mathrm{M} \Omega$ when filled with $\mathrm{KCl}$ based intracellular solution containing the following (in $\mathrm{mM}$ ): $136 \mathrm{KCl}, 17.8$ HEPES, 1 EGTA, $4.6 \mathrm{MgCl}_{2}, 4$ ATP- $\mathrm{Na}_{2}, 0.3$ GTP-Na 2,12 creatine phosphate, and $50 \mathrm{U} / \mathrm{ml}$ phosphocreatine kinase ( $>300 \mathrm{mOsm}$; $\mathrm{pH}$ 7.4). Neurons transduced with viruses were visually identified by RFP and GFP expression before recordings. Series resistance was compensated by $70 \%$ and monitored over the course of each recording. The recordings were analyzed offline using Axograph X Version 1.5.4 (Axograph Scientific).

Glutamatergic hippocampal neurons were clamped at $-70 \mathrm{mV}$ at steady state and EPSCs were evoked by a $2 \mathrm{~ms}$ depolarizing step to $0 \mathrm{mV}$. EPSCs were then recorded in the presence of $3 \mu \mathrm{M}$ NBQX (Tocris Bioscience), which is an AMPA receptor antagonist. The averages of the traces recorded in the presence of NBQX were subtracted by using subtraction function in AxographX from the average of the traces recorded in the external solution without NBQX to cancel stimulus artifacts. Spontaneous release was determined by monitoring miniature EPSCs (mEPSCs) for 30-60 s at $-70 \mathrm{mV}$. To correct false-positive events, mEPSCs were again recorded in the presence of $3 \mu \mathrm{M}$ NBQX. The recorded traces were filtered at $1 \mathrm{kHz}$ and mEPSCs were detected with a template function in Axograph X. The readily releasable pool (RRP) size was determined by a $5 \mathrm{~s}$ application of extracellular solution that additionally contained $500 \mathrm{~mm}$ sucrose (Rosenmund and Stevens, 1996). The solutions were exchanged with a fast microperfusion device (SF-77B; Warner Instruments).

High-pressure freezing and electron microscopy. Hippocampal neurons were plated onto $6 \mathrm{~mm}$ sapphire disks with a density of $50 \times 10^{3}$ to $100 \times$ $10^{3}$ cells. The neurons were coinfected at DIV 2 with viruses to express Cre recombinase in combination with control viruses or with viruses to express Stx1A ${ }^{\mathrm{WT}}$ or Stx1 ${ }^{\mathrm{AV}}$. At DIV 12-14, the samples were mounted into a sandwich support with extracellular solution containing $15 \% \mathrm{Fi}$ coll (Sigma-Aldrich) to avoid ice crystal damage and frozen with an HPM 100 Leica high-pressure freezer under a pressure of 2100 bar. Samples were then transferred into cryovials containing $1 \%$ glutaraldehyde, $1 \%$ osmium tetroxide, $1 \%$ Milli-Q water (Millipore) in anhydrous acetone and processed in an AFS2 automated freeze-substitution device (Leica) followed by a temperature ramp from $-90^{\circ} \mathrm{C}\left(8^{\circ} \mathrm{C} / \mathrm{h}\right)$ to $-50^{\circ} \mathrm{C}$, from 
$-50^{\circ} \mathrm{C}\left(6^{\circ} \mathrm{C} / \mathrm{h}\right)$ to $-20^{\circ} \mathrm{C}$, constant temperature for $12 \mathrm{~h}$ at $-20^{\circ} \mathrm{C}$, and a ramp from $-20^{\circ} \mathrm{C}\left(5^{\circ} \mathrm{C} / \mathrm{h}\right)$ to $20^{\circ} \mathrm{C}$. After the freeze-substitution step, samples were contrasted with $0.1 \%$ uranyl acetate for $1 \mathrm{~h}$ and embedded in epoxy epon 812 . Finally, samples were placed into capsules filled with pure epoxy epon 812 and further polymerized for $48 \mathrm{~h}$ at $60^{\circ} \mathrm{C}$. Areas of $\sim 200 \mu \mathrm{m}^{2}$ containing cells were randomly selected and 40-nm-thick sections were obtained with a Leica Ultracut and collected on Formvarcoated $(0.5 \%)$ 200-mesh copper grids. Those sections were further examined on a Tecnai G20 electron microscope operated at $80-200 \mathrm{keV}$ (FEI). Electron micrographs $(2048 \times 2048$ pixels $)$ were collected blindly using a Velata camera (FEI) with an $89,000 \times$ magnification (pixel size $0.57 \mathrm{~nm}$ ). Data were analyzed blindly using an analysis program developed for ImageJ and MATLAB (Watanabe et al., 2013). Active zones (AZs) were defined by a corresponding postsynaptic density attached to the presynaptic bouton and docked vesicles were defined as those in direct contact with the plasma membrane. Synaptic vesicle distribution was analyzed by calculating the shortest distance from each vesicle to the presynaptic active zone.

Statistical analysis. Data are presented as means \pm SEM. For statistical analysis, data from two groups were subjected to Student's two-tailed $t$ test and data from more than two groups were subjected to one-way ANOVA followed by Tukey's post hoc test with GraphPad Prism 5.0b. $p$-values $>0.05$ were considered insignificant.

\section{Results \\ Deletion of both Stx1 isoforms Stx1A and Stx1B is prenatally lethal}

The neuronal t-SNARE protein Stx1 is essential for neuronal maintenance and neurotransmitter release in worms and flies (Schulze et al., 1995; Schulze and Bellen, 1996; Burgess et al., 1997); however, dissecting these functions in mice has been unfeasible due to the functional redundancy of Stx1 isoforms Stx1A and Stx1B (Fujiwara et al., 2006; Gerber et al., 2008; Wu et al., 2015). To eliminate the reciprocal compensatory effects of Stx1A and Stx1B and to thereby study the essential functions of Stx1, we established an Stx1A/1B DKO mouse line by interbreeding heterozygous Stx1B Het $(+/-)$ mice with homozygous Stx1A $\mathrm{KO}(-/-)$ mice, which were generated as described previously (Gerber et al., 2008; Wu et al., 2015). Because Stx1A KO mice reportedly display normal development, life span, and neurotransmitter release (Fujiwara et al., 2006; Gerber et al., 2008; Arancillo et al., 2013), all the mice used in this study were held on Stx1A-null background.

Similar to a previous report (Mishima et al., 2014), deletion of both Stx1 isoforms resulted in embryonic lethality. In addition, we observed gross morphological abnormalities at E16-E18 (Fig. $1 A$ ), at which state the Stx1A/1B DKO embryos were already distinguishable by a hunched back and distorted body posture visible to the naked eye (Fig. $1 A$ ), a phenotype shared by the other SNARE KO and Munc13-1/2 DKO mutants (Schoch et al., 2001; Washbourne et al., 2002; Varoqueaux et al., 2005). Loss of Stx1B was confirmed by Western blot analysis of whole-brain lysates derived from E18 mice (Fig. 1B). To assess the impact of Stx1 deletion on brain assembly, we examined major brain structures by Nissl staining of the brain tissues of Stx1A KO, Stx1B Het/ Stx1A KO, and Stx1A/1B DKO embryos dissected at E16 and E18 (Fig. 1C). Based on the observation of no significant differences between Nissl-stained brain tissues from Stx1A KO and Stx1B Het/Stx1A KO, data from these groups were pooled as control. Complete deletion of Stx 1 led to partial cell loss in the cerebellum and brainstem at E16 (Fig. 1C). At E18, cell loss in the brainstem and the cerebellum was even more substantial and became visible in the cerebral cortex and hypothalamus (Fig. 1C), indicating that the regular brain structures are first formed but lost at later embryonic stages: a similar phenotype to Munc18-1 KO mice (Ver- hage et al., 2000). These findings indicate that Stx1 function is essential during embryonic brain development.

Being high-affinity interaction partners (Hata et al., 1993), Munc18-1 and Stx1 regulate one another's expression levels either by serving as chaperones (Rowe et al., 1999, 2001), by assisting the proper localization, or by enhancing the stability of one another (Toonen et al., 2005). It has been shown that Munc18-1 deletion results in an $\sim 70 \%$ reduction in both Stx1A and Stx1B levels (Toonen et al., 2005), whereas a $\sim 25 \%$ reduction in Munc18-1 protein level is observed either when Stx1B is deleted (Mishima et al., 2014; Wu et al., 2015) or expressed at severely low levels (Gerber et al., 2008; Arancillo et al., 2013; Zhou et al., 2013). Conversely, deletion of Stx1A alone has no effect on Munc18-1 expression, most likely due to the compensatory action of Stx1B (Fujiwara et al., 2006; Mishima et al., 2014). To address whether the deletion of both isoforms further enhances the decrease in Munc18-1 levels, we performed Western blot analysis using whole-brain lysates derived from E18 mice. Munc18-1 expression was $\sim 40 \%$ reduced in the Stx1A/1B DKO embryos compared with the Stx1A KO (Fig. 1D). Moreover, unlike in Stx1A, Stx1B, or Munc18-1 KO mice (Verhage et al., 2000; Toonen et al., 2005; Fujiwara et al., 2006; Wu et al., 2015), we observed significant reductions from $\sim 20 \%$ to $60 \%$ in expression levels of other tested exocytotic proteins SNAP-25, Syb2, synaptotagmin-1 (Syt1), Munc13-1, and complexin-1 (Cpx1), as well as the early endosomal protein Rab5, in Stx1A/1B DKO embryos (Fig. 1D), suggesting that widespread cell loss and/ or synaptic loss in the embryonic mouse brains results in reduced levels of synaptic proteins or that the removal of Stx1 downregulates the expression of other proteins involved in secretion.

\section{Stx1 is required for neuronal maintenance in vitro}

To investigate whether Stx1A/1B DKO neurons can survive in vitro, dissociated cortical neurons from E16 mice were cultured at high density (see Materials and Methods). The neurons were cocultured with astrocyte feeder layers. The astrocytes were all derived from WT newborn mice to provide comparable trophic support to both Stx1A/1B DKO and control neurons. At DIV 1, $\sim 60 \%$ of control Stx1A KO neurons initially plated were present in the culture, the cell density remained largely unchanged for 3 weeks (Fig. 2A), and the neurons appeared healthy overall (Fig. $2 C)$. On the contrary, the number of surviving Stx1A/1B DKO neurons dropped to $17 \%$ within $1 \mathrm{~d}$ and reached $\sim 1 \%$ at DIV 4 , suggesting that $\mathrm{Stx} 1$ is also required for neuronal maintenance in vitro (Fig. 2A). No Stx1A/1B DKO neurons survived at DIV 22 (Fig. 2A). Even though deletion of Stx 1 resulted in severe cell loss, the remaining neurons at DIV 2 and 4 could form dendritic processes as reflected by positive immunofluorescence labeling for MAP2 (Fig. 2B), which indicates that Stx1A/1B DKO neurons could still differentiate. However, the intensity of MAP2 signal and the complexity of dendritic branching appeared reduced in Stx1A/1B DKO neurons compared with the Stx1A KO neurons (Fig. 2B).

To restore the neuronal viability in vitro, we reintroduced either Stx1A or Stx1B expression in Stx1A/1B DKO neurons by infecting with lentiviruses immediately after culturing (DIV 0). The control Stx1A KO group was infected with lentiviruses to induce expression of only NLS-GFP to monitor the effect of virus load on the viability of neurons. Exogenous expression of Stx1A or Stx1B rescued the Stx1A/1B DKO neurons against cell death by $17 \%$ and $11 \%$, respectively, compared with the Stx1A KO neurons (Fig. 2C), with the low rescue efficiency reflecting the short time window of the survival of Stx1A/1B DKO neurons. Immu- 
A

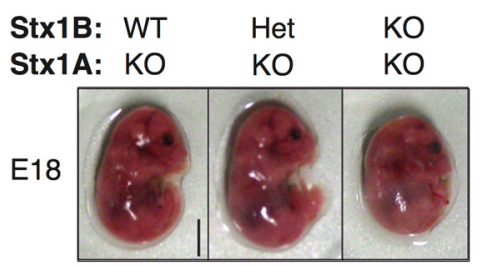

B

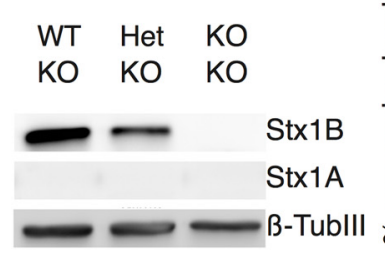

Stx1B: Het KO

Stx1A: KO KO

C
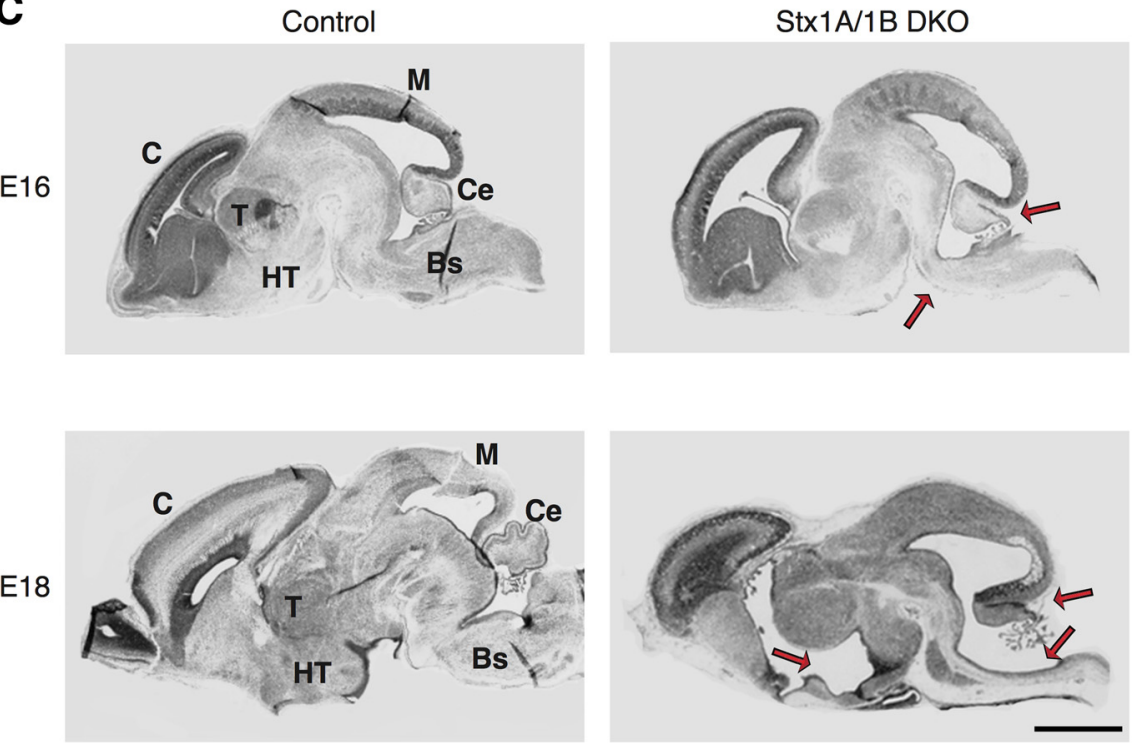

D
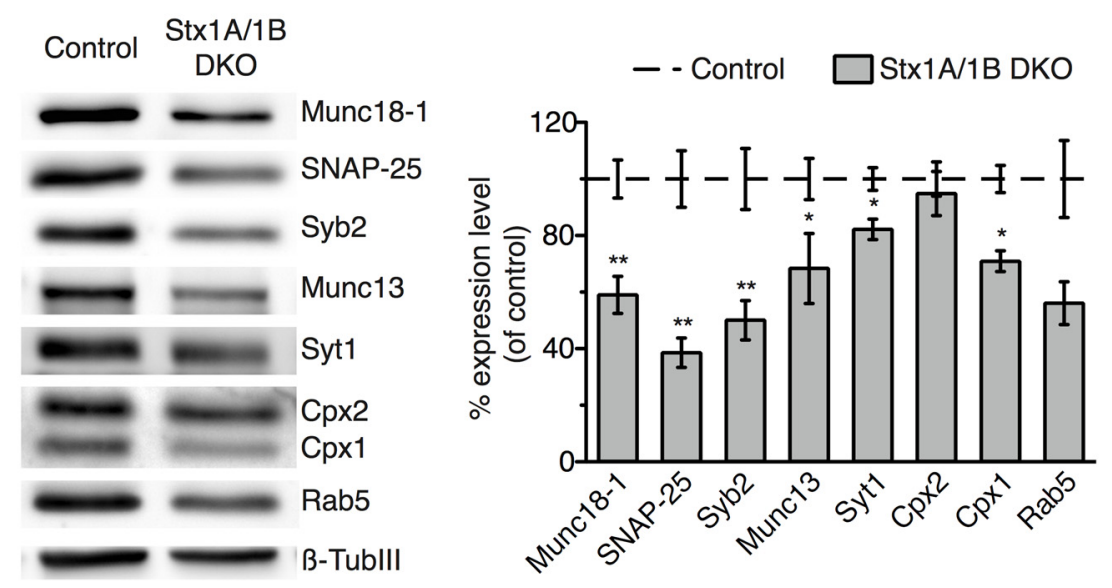

Figure 1. Deletion of both Stx1 isoforms Stx1A and Stx1B is prenatally lethal. $\boldsymbol{A}$, Stx1A/1B DK0 mice (right) show gross morphological abnormalities at E18 compared with Stx1A K0 (left) and Stx1B Het/Stx1A K0 (middle). Scale bar, $1 \mathrm{~cm}$. $\boldsymbol{B}$, Example immunoblot showing Stx1A and Stx1B protein levels (left) and summary graph of quantification of Stx1B protein levels (right) in the whole-brain lysates obtained from E18 control and Stx1A/1B DK0 mice. $n=6$ animals for each genotype. C, Example Nissl-stained sagittal brain sections from control (left) and Stx1A/1B DK0 (right) mice at E16 (top) and E18 (bottom). Arrows show the brain structures with excessive cell loss. Bs, Brainstem; C, cortex; Ce, cerebellum; HT, hypothalamus; M, midbrain; T, thalamus. Scale bar, $1 \mathrm{~mm} . n=3$ animals for each genotype. $\boldsymbol{D}$, Example immunoblot (left) and summary graph of quantification (right) of Munc18-1, SNAP-25, Syb-2, Munc13, synaptotagmin-1 (Syt-1), complexin-2 (Cpx2), Cpx1, and Rab5 protein levels in the whole-brain lysates obtained from E18 mice. $n=5$ animals for each genotype. Bar graphs show means \pm SEM. Statistical analysis was applied by one-way ANOVA followed by Tukey's post hoc test in $\boldsymbol{B}$ and Student's $t$ test in $\boldsymbol{D} ;{ }^{*} p \leq 0.05,{ }^{* *} p \leq 0.01 ;{ }^{* * *} p \leq 0.001$ nofluorescence analysis of the exogenously expressed Stx1A or Stx1B protein levels, as normalized to the immunofluorescence signal of presynaptic active zone protein Bassoon, revealed similar or slightly elevated expression levels of Stx1A or Stx1B compared with controls (Fig. $2 D$ ). The rapid death caused by loss of Stx 1 and the revival of a fraction of Stx1A/1B DKO neurons by exogenous expression of Stx1A or Stx1B signify the absolute requirement of Stxl, whereas the similar rescue efficiencies of Stx1A and Stx1B manifest the functional redundancy between Stx1A and Stx1B in terms of neuronal maintenance.

\section{Loss of Stx1 leads to an earlier and} more severe neuronal lethality than loss of Munc18-1

The severe cytotoxicity caused by loss of Stx1 is reminiscent of Munc18-1 deficiency (Verhage et al., 2000; Heeroma et al., 2004). However, loss of Munc18-1 KO neurons could be delayed up to DIV 10, when trophic support was provided (Heeroma et al., 2004), suggesting that loss of Stx1 might be more deleterious than loss of Munc18-1. To compare the severity of cell loss in Stx1A/1B DKO and Munc18-1 $\mathrm{KO}$ neurons directly in the same culture conditions, we obtained cortical cultures from E16 Munc18-1 WT and KO mouse brains. Similar to the Stx1A KO neurons (Fig. $2 A$ ), $\sim 55 \%$ of WT neurons initially plated were present in the culture at DIV 1 and the number remained unchanged for the next 3 weeks (Fig. 3A). Conversely, at DIV 1, $\sim 40 \%$ of Munc18-1 KO neurons were still alive and the remaining Munc18-1 KO neurons were gradually lost within $10 \mathrm{~d}$ (Fig. 3A). In addition, exogenous expression of Munc18-1 in Munc18-1 KO neurons showed an 70\% rescue efficiency in neuronal survival (Fig. $3 B)$. The slower rate of cell loss and the higher rescue capacity of lentiviral expression of Munc18-1 suggest that loss of Munc18-1 is less toxic than loss of Stx1 in neurons.

The similarity between the Stx1A/1B DKO and Munc18-1 KO phenotypes demonstrates the importance of these proteins for neuronal maintenance, most likely as a complex. However, severe reduction in Stx1 expression and/or its destabilization in the absence of Munc18-1 (Toonen et al., 2005) raises the possibility that the lethality of Munc18-1 KO neurons might be a direct consequence of low Stx1 levels. Similarly, impairment in Munc18-1 expression might be the cause of lethality of the Stx1A/1B DKO neurons. 
A

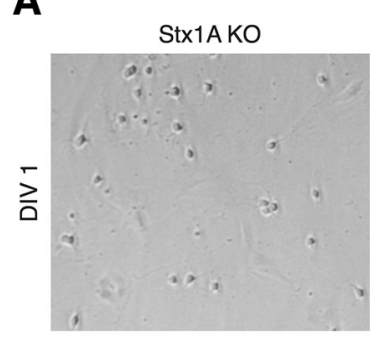

B
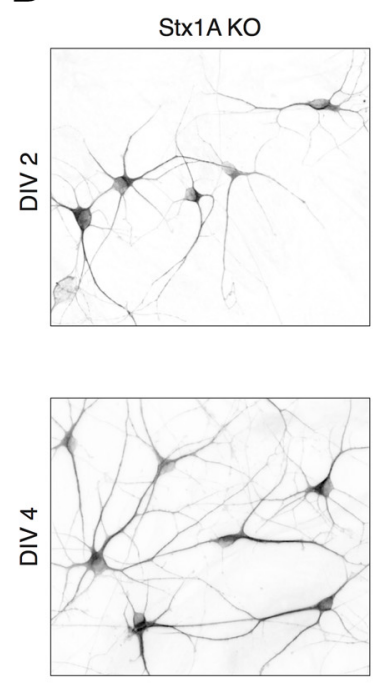

D

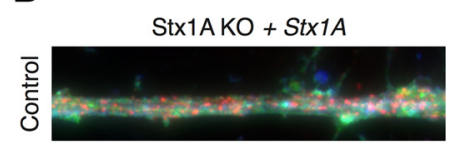

Stx1A/1B DKO+Stx1A

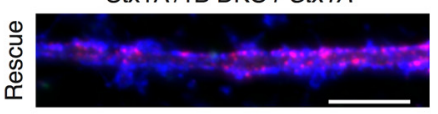

Stx1A/1B DKO

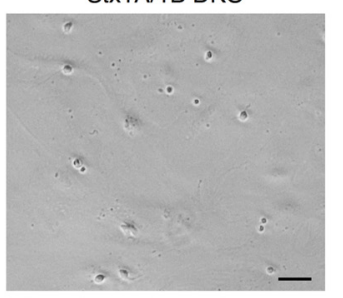

Stx1A/1B DKO
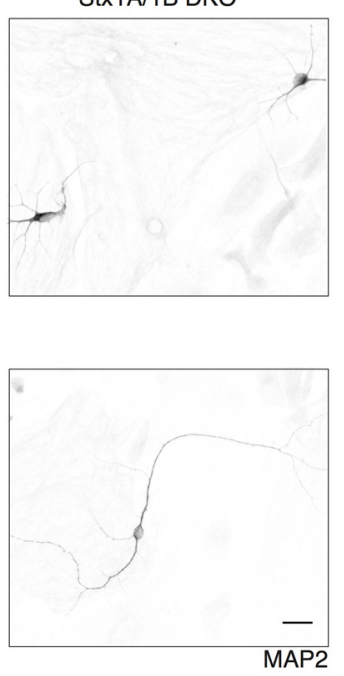

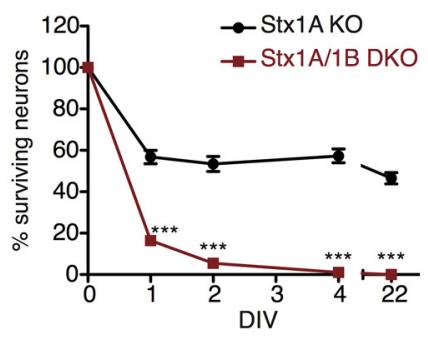

C

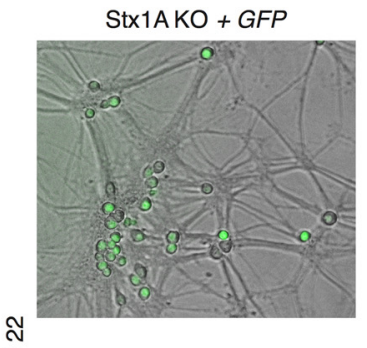

$\geq$

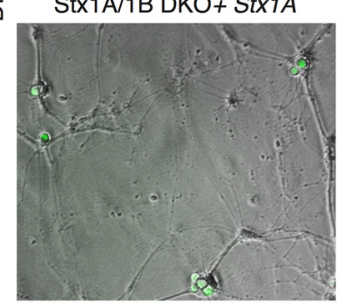

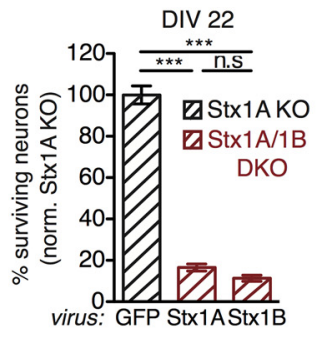

$\mathrm{Stx1A} / 1 \mathrm{~B} \mathrm{DKO}+\mathrm{Stx} 1 \mathrm{~B}$

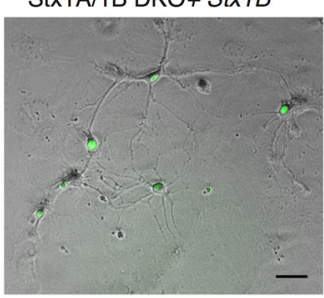

Stx1AKO + GFP

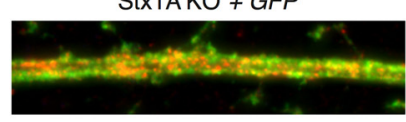

Stx1A/1B DKO + Stx1B

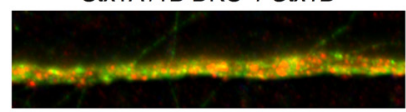

Bassoon;Stx1A;Stx1B
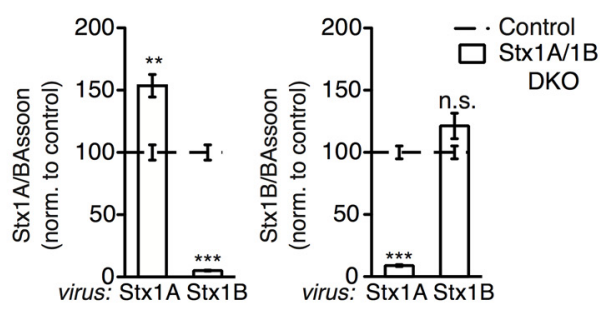

Figure 2. Stx1 is required for neuronal maintenance in vitro. $\boldsymbol{A}$, Example images of cultured Stx1A K0 and Stx1A/1B DK0 cortical neurons at DIV 1 (left) and summary graph of percentage of surviving neurons in the cultures at DIV 1,2,4, and 22 (right). $n=58-60$ images at each time point from three independent cultures. Scale bar, $50 \mu m$. $B$, Example images of immunofluorescence labeling for MAP2 in cultured Stx1A K0 and Stx1A/1B DK0 cortical neurons at DIV 2 (top) and DIV 4 (bottom). Scale bar, $20 \mu \mathrm{m}$. C, Example images (left) and summary graph of percentage (right) of surviving neurons at DIV 22 in the Stx1A K0 cultures infected with viruses expressing only NLS-GFP and in the Stx1A/1B DK0 cultures infected with viruses expressing either Stx1A or Stx $1 B$. $n=$ 58 - 60 images from three independent cultures. D, Example images (left) and summary graphs (right) of immunofluorescence labeling for Stx1A (blue) and Stx $1 B$ (green) in control and rescued neurons at DIV 22. The average intensities of Stx1A or Stx1B immunofluorescence signals were analyzed at compartments positive for Bassoon immunofluorescence signal (red). Stx1A K0 neurons infected with viruses to express only NLS-GFP or Stx1B were used as controls (dashed lines). $n=42-49$ images from three independent cultures. Data points in $\boldsymbol{A}$ and bar graphs in $\boldsymbol{C}$ and $\boldsymbol{D}$ show means \pm SEM. Statistical analysis was applied by Student's $t$ test in $\boldsymbol{A}$ and by one-way ANOVA followed by Tukey's post hoc test in $\boldsymbol{C}$ and $\boldsymbol{D}$; ${ }^{* * *} p \leq 0.001$.

To test whether the decreased Stx 1 expression level is the primary factor for the death of Munc18-1 KO neurons and vice versa, we used a cross-rescue approach. For this purpose, we overexpressed either Stx1A or Stx1B in Munc18-1 KO neurons and Munc18-1 in Stx1A/1B DKO neurons in vitro. Exogenous expression of Munc18-1 displayed no significant effects on the survival of Stx1A/1B DKO neurons at DIV 4 (Fig. 3C), indicating that increasing Munc18-1 protein levels is not sufficient to rescue Stx1A/1B DKO neurons against cell death. Conversely, expression of either Stx1A or Stx1B in Munc18-1 KO neurons resulted in prolonged survival (Fig. $3 D, E$ ). The number of surviving Munc18-1 KO neurons increased from $\sim 5 \%$ to $\sim 15 \%$ at DIV 6 and from $\sim 0.5 \%$ to $\sim 3 \%$ at DIV 10 , when Stx1A was overexpressed. Interestingly, the rescue efficiency of Stx1B in Munc18-1 KO neurons was significantly higher than the rescue efficiency of Stx1A. At DIV 6, 25\% of Munc18-1 KO neurons were still present in the culture and this number was reduced only to $\sim 10 \%$ at DIV 10, when Stx1B was overexpressed (Fig. $3 D, E$ ). We also noticed that loss of Munc18-1 led to a more severe reduction in Stx1B signal than in Stx1A signal as revealed by Western blot analysis of whole-brain lysates derived from E16 Munc18-1 WT and KO animals (Fig. 3F). This result, together with the observation of a better rescue efficiency of Stx1B in Munc18-1 KO neurons, may suggest that Munc18-1 interacts more strongly with Stx1B than with Stx1A. In addition, although it is clear that both Munc18-1 and Stx1 are absolutely required for cell survival, the more cytotoxic effect of loss of Stx1 than loss of Munc18-1 indicates that Stx1, but not Munc18-1, is absolutely essential for neuronal survival. Alternatively, both Stx1 and Munc18-1 have equally important functions in neuronal sur- 
A
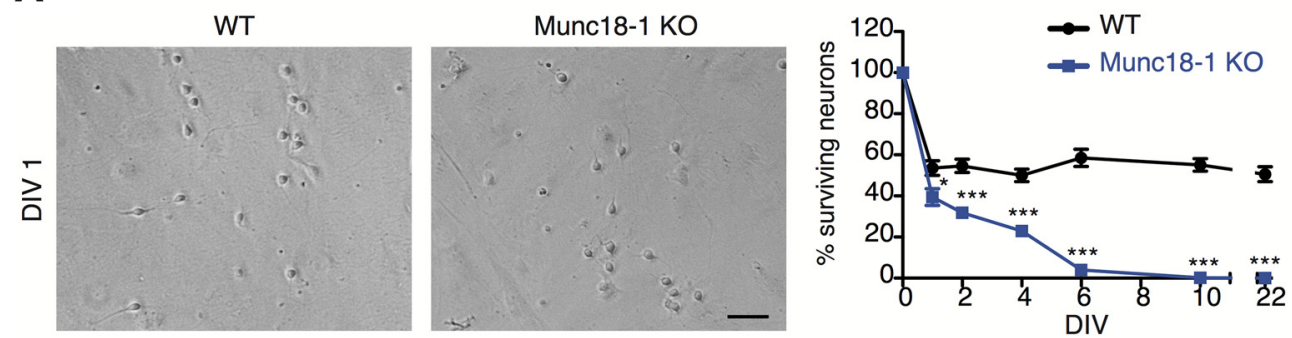

B
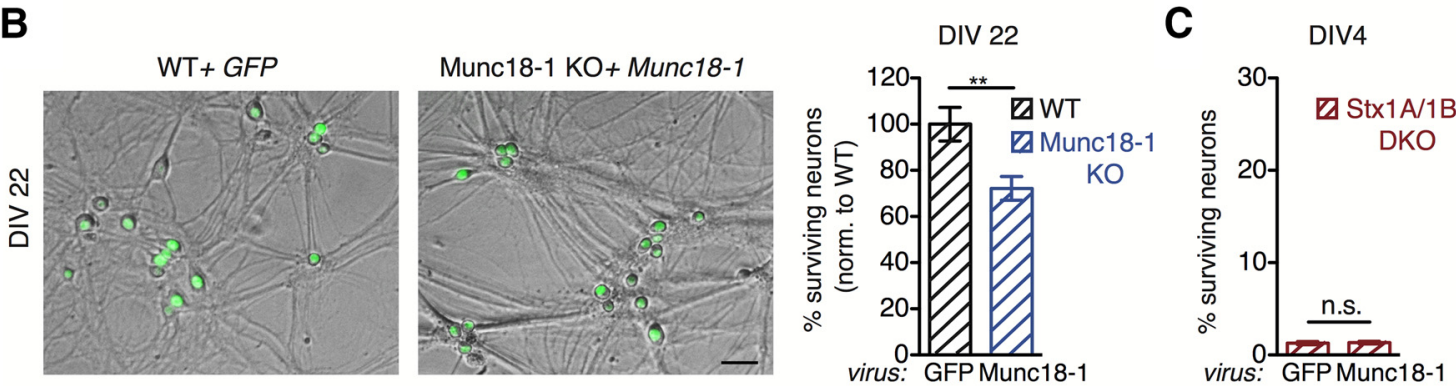

D
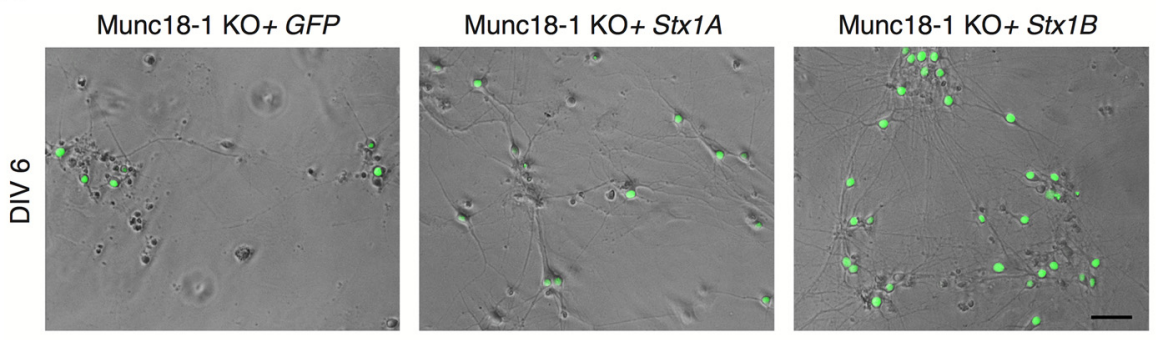

E

DIV6

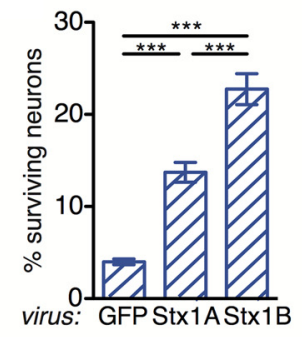

DIV10

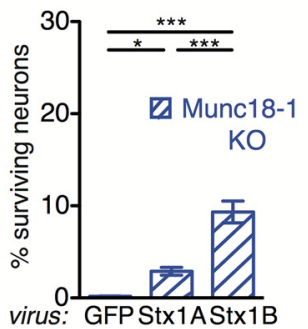

F

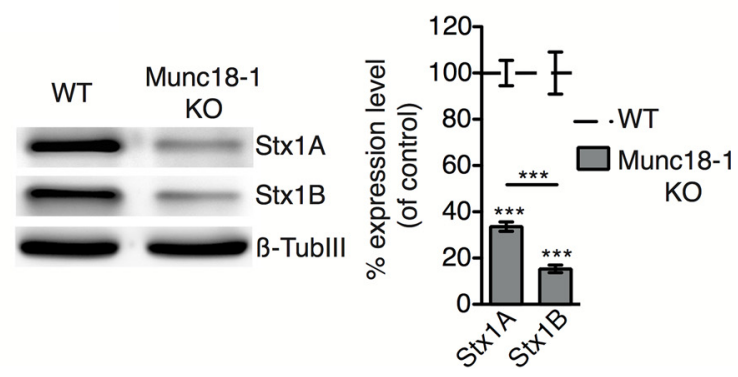

Figure 3. Loss of Stx1 leads to an earlier and more severe neuronal lethality than loss of Munc18-1. $A$, Example images of cultured WT and Munc18-1 K0 cortical neurons at DIV 1 (left) and summary graph of percentage of surviving neurons in the cultures at DIV 1-10 and at DIV 22 (right). $n=58-60$ images from three independent cultures. $\boldsymbol{B}$, Example images (left) and summary graph of percentage (right) of surviving neurons at DIV 22 in the WT culture infected with viruses to express only NLS-GFP and in the Munc18-1 K0 cultures infected with viruses to express Munc18-1. $n=60$ images from three independent cultures. C, Summary graph of percentage of surviving Stx1A/1B DKO neurons at DIV 4 infected with viruses to express only NLS-GFP or Munc18-1. D, Example images of surviving Munc18-1 KO neurons at DIV 6 infected with viruses to express only NLS-GFP, Stx1A, or Stx1B. E, Summary graphs of percentage of Munc18-1 K0 neurons expressing only NLS-GFP, Stx1A, or Stx1B at DIV 6 and 10. $n=60$ images from three independent cultures. $F$, Example immunoblot (left) and summary graph (right) showing Stx1A and Stx1B protein levels in whole-brain lysates obtained from E16 WT and Munc18-1 K0 animals. $n=5$ animals for each genotype. Scale bars in A, B, and D, $50 \mu \mathrm{m}$. Data points in $\boldsymbol{A}$ and bar graphs in $\boldsymbol{B}, \boldsymbol{C}, \boldsymbol{E}$, and $\boldsymbol{F}$ show means \pm SEM. Statistical analysis was applied by Student's test in $\boldsymbol{A}-\boldsymbol{C}$ and $\boldsymbol{F}$ and by one-way ANOVA followed by Tukey's post hoc test in $\boldsymbol{E}$; ${ }^{*} p \leq 0.05 ;{ }^{* *} p \leq 0.01$; ${ }^{* * *} p \leq 0.001$.

vival, but another Munc18 isoform, such as Munc18-2, which has been shown to bind to Stx1 (Hata and Südhof, 1995; Rowe et al., 2001; Gulyás-Kovács et al., 2007), partially supports cell viability.

Stxl is essential for neuronal maintenance, not only at early developmental stages, but also after maturation in vitro

The immature death of Stx1A/1B DKO neurons not only restrains the investigation of the Stx 1 requirement for neuronal maintenance to the developing neurons, but also renders the studies of the impact of Stx1 loss on synaptic transmission impracticable. Is the Stx 1 requirement for neuronal maintenance confined to development or do neurons perpetually need Stx 1 function for viability also after maturation? By using a Cre/LoxP recombination system, we generated an Stx1A/1B conditional DKO ( $\mathrm{cDKO}$ ) mouse line in which the Stx1B gene was flanked by two LoxP sites on an Stx1A-null background $\left(\mathrm{Stx} 1 \mathrm{~B}^{\mathrm{FL} / \mathrm{FL}} / \mathrm{Stx} 1 \mathrm{~A} \mathrm{KO}\right)$ and thereby bypassed the premature death of Stx1-deficient neurons. High-density hippocampal cultures were prepared from newborn homozygous Stx1A/1B cDKO mice and Stx1B was removed in vitro by Cre recombinase 
A
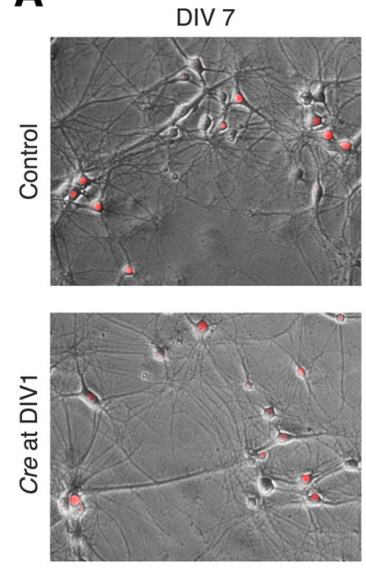

B

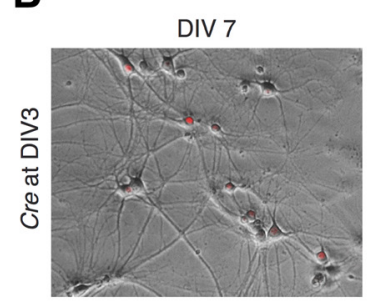

C

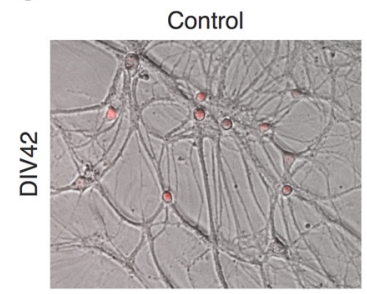

DIV 13
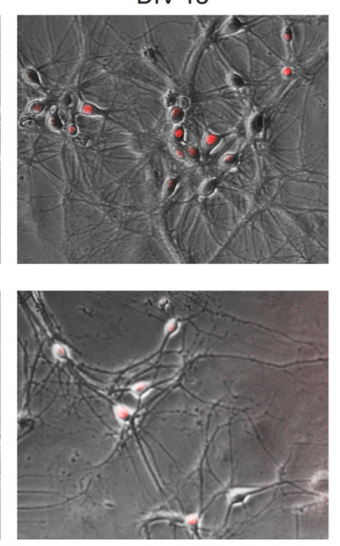

DIV 13

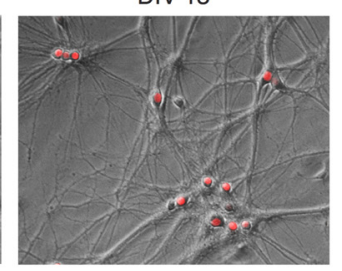

Cre at DIV3

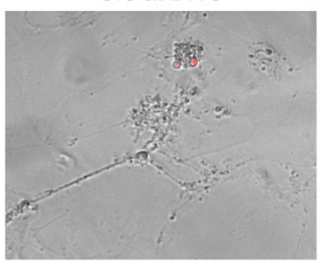

DIV 23
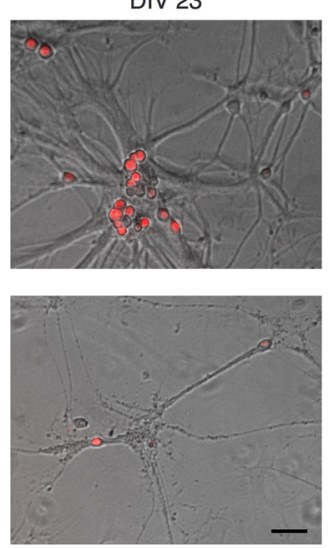

DIV 23

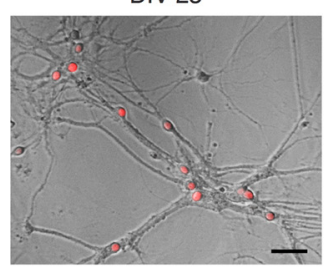

Cre at DIV5

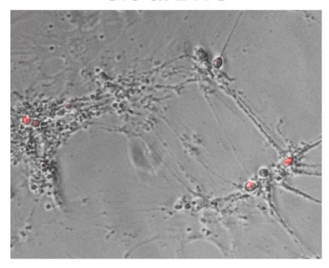

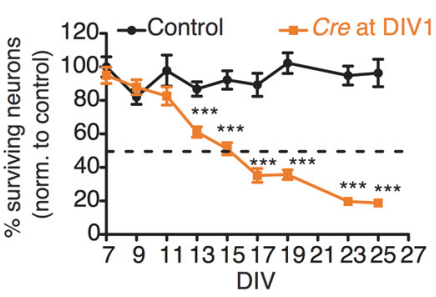

DIV

Figure 4. Stx1 is essential for neuronal maintenance after maturation in vitro. $A$, Example images (left) and summary graph of percentage (right) of surviving cultured hippocampal neurons derived from P0 - P2 Stx 1B FL/FL/Stx $1 A$ KO animals. Stx $1 \mathrm{~A} / 1 \mathrm{~B} C \mathrm{CDK} 0$ neurons were generated by infection at DIV 1 with viruses to express Cre recombinase. Cultures infected with viruses to express only NLS-RFP were used as a control. $n=40$ images from two independent cultures for DIV 11, 15, 17, 19, 21, and 25. $n=59-62$ from three independent cultures for DIV 7,9, 13, and 23. $B$, Example images of Stx1A/1B CDKO cultures generated by infection at DIV 3 with viruses to express Cre recombinase (left) and summary graph of percentage of surviving control and Stx1A/1B cDK0 neurons infected at DIV 3, 5 , and 7 with viruses to express (re recombinase (right). $n=39-40$ images from two independent cultures for DIV 11, 15, 19, 27, 33, 39, and 42 and $n=58-60$ from three independent cultures for DIV 7 and 23. Scale bar, $50 \mu \mathrm{m}$. C, Example images of control and Stx $1 \mathrm{~A} / 1 \mathrm{~B}$ cDK0 cultures at DIV 42 . Scale bars, $50 \mu \mathrm{m}$. Data points show means \pm SEM. Statistical analysis was applied by Student's $t$ test; ${ }^{*} p \leq 0.05 ;{ }^{* *} p \leq 0.01 ;{ }^{* * *} p \leq 0.001$.

expression induced by lentiviral infection at DIV 1. The control neurons were of the same genotype (Stx1B $\left.{ }^{\mathrm{FL} / \mathrm{FL}} / \mathrm{Stx} 1 \mathrm{~A} \mathrm{KO}\right)$, but were transduced with viruses expressing only the reporter construct NLSRFP. The number of surviving neurons was determined between DIV 7 and 25 and normalized to the number of neurons of the control group at DIV 7. The number of the control neurons and the number of the Stx1A/1B cDKO neurons infected with viruses at DIV 1 to express Cre recombinase were comparable between DIV 7 and 11 (Fig. 4A). However, we observed a gradual reduction of viable Stx1A/1B cDKO neurons starting at DIV 13 and the neuronal viability reached $50 \%$ at DIV 15 , whereas the number of control neurons did not show any significant differences between DIV 7 and 25 (Fig. 4A), showing Stx1 dependency for neuronal maintenance after development.

To further assess the impact of Stx1 loss on neuronal maintenance at later stages after neuronal maturation is accomplished, Cre recombinase expression was induced by infection at DIV 3, 5, and 7, and subsequently the neuronal viability was monitored up to DIV 42. The delayed induction of Cre recombinase expression and thus the delayed Stx1B turnover ultimately led again to neuronal lethality but with a delayed onset. The viability declined to $50 \%$ at DIV 23, 33, and 39 for neurons that were transduced with Cre recombinase at DIV 3, 5, and 7, respectively (Fig. $4 B$ ). The time between genetic removal of Stx1 and cell loss was in the range of 2-3 weeks (Fig. $4 A, B)$, suggestive of a slow Stx1 turnover and/or that only a severe drop in Stx1 levels leads to cell death, which is consistent with the findings from previous Stx1 hypomorphic models (Arancillo et al., 2013). The severity of the structural defects at DIV 42 appeared more substantial in the groups infected with Cre recombinase at earlier time points (Fig. 4C). Altogether, these data demonstrate that Stxl is essential for neuronal maintenance, not only at early developmental stages, but throughout the lifespan of a neuron.

\section{Stx 1 is required for vesicle priming and fusion}

The in vivo and in vitro lethality observed in Stx1-deficient neurons demonstrate that Stx1 is essential for neuronal maintenance during development and after maturation; however, it also obscures the investigation of Stx1's functions in neurotransmitter 
A
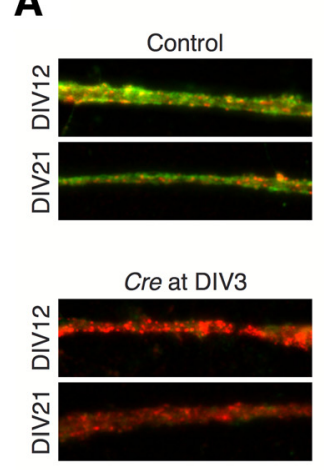

C

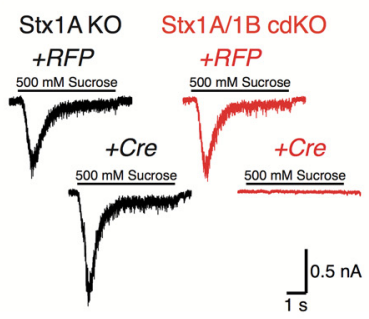

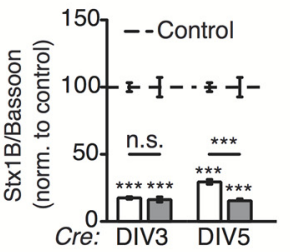

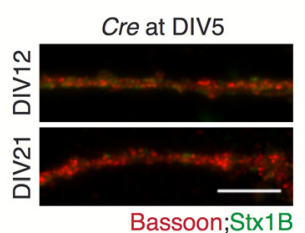

Bassoon;Stx1B $\square$ DIV12
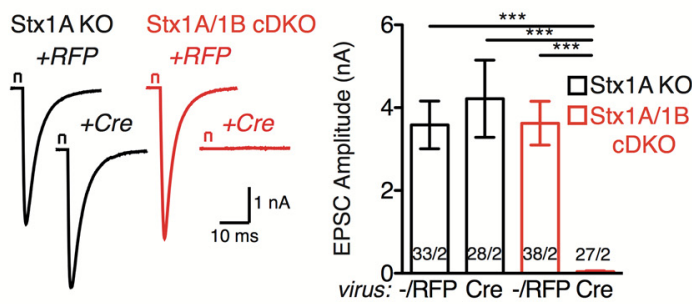

D
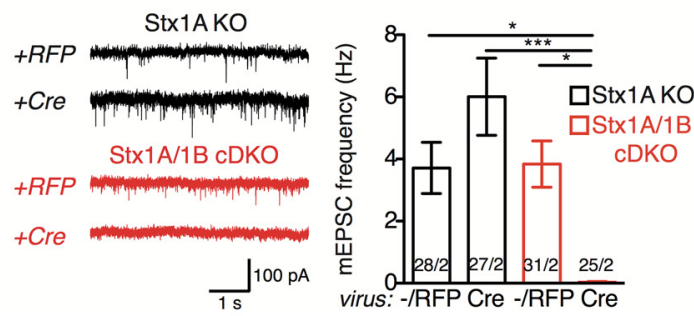

Figure 5. Stx1 is required for vesicle priming and fusion. $A$, Example images (top left and bottom) and summary graph (top right) of immunofluorescence labeling for Stx $1 \mathrm{~B}$ ( $\mathrm{green}$ ) in control and in Stx1A/1B cDK0 neurons infected at DIV 3 or 5 with viruses to express (re recombinase. The average intensities of Stx1B immunofluorescence signals were measured at compartments positive for Bassoon immunofluorescence signal (red) and the values were normalized to the values obtained from control neurons (dashed lines). $n=24-41$ images from two independent cultures. $\boldsymbol{B}$, Example traces (left) and summary graph (right) of EPSCs evoked by a 2 ms depolarization in autaptic neurons derived from P0 -P2 Stx1A K0 and Stx1A/1B cDK0 mice. The neurons were infected at DIV 3 with viruses to express Cre recombinase. C, Example traces (left) and summary graph (right) of readily releasable pool in Stx $1 A$ K0 and Stx $1 A / 1 B$ cDK0 neurons determined by 500 mm sucrose application for 5 s. D, Example traces (left) and summary graph (right) of mEPSCs in Stx1A K0 and Stx1A/1B CDK0 neurons recorded at holding potential -70 mV. Bar graphs show means \pm SEM. Statistical analysis was applied by one-way ANOVA followed by Tukey's post hoc test; ${ }^{*} p \leq 0.05,{ }^{* *} p \leq 0.01,{ }^{* * *} p \leq 0.001$. Cell numbers and the number of independent cultures are presented in the graphs in $\boldsymbol{B}-\boldsymbol{D}$. Artifacts and/or action potentials are blanked in $\boldsymbol{B}$ and $\boldsymbol{C}$. The traces were filtered at $1 \mathrm{kHz}$ in $\boldsymbol{D}$.

release such that the impacts of Stx1 deficiency on neurotransmission in mammalian neurons could be determined only by severe reduction of Stx1B expression on a Stx1A-null background (Arancillo et al., 2013; Zhou et al., 2013). To find a time window for electrophysiological examination at which the neurons are mature, yet intact and completely devoid of Stx1, we monitored Stx1B protein levels in Stx1A/1B cDKO neurons, which were infected at DIV 3 or 5 with viruses to express Cre recombinase and fixed at DIV 12 and 21 (Fig. 5A). Immunofluorescence labeling in both Stx1A/1B cDKO groups expressing Cre recombinase revealed Stx1B signals at a level of $15-30 \%$ compared with the control neurons at DIV 12, whereas at DIV 21, no further reduction in Stx1B signal was observed in Stx1A/1B cDKO neurons infected at DIV 3 (Fig. 5A). This suggests that the values obtained in this group for Stx1B signal at DIV 12 represent background (Fig. 5A). We therefore chose infection at DIV 3 to express Cre recombinase in Stx1A/1B cDKO neurons to eliminate Stx1B because, at DIV 12, these neurons were still alive, appeared intact, and yet Stx1 was completely absent (Figs. 4B, 5A).

To evaluate the impact of loss of Stx1 on neurotransmitter release, we recorded $\mathrm{Ca}^{2+}$-evoked release, spontaneous release, and hypertonic sucrose solution-evoked release from autaptic hippocampal cultures of Stx1A/1B cDKO neurons compared with WT-like Stx1A KO neurons. The control neurons, namely the Stx1A KO neurons expressing either the reporter NLS-RFP or Cre recombinase, as well as Stx1A/1B cDKO neurons expressing only NLS-RFP, produced robust and comparable EPSCs with an average peak amplitude of $\sim 4 \mathrm{nA}$ (Fig. $5 B$ ). Conversely, loss of both Stxl isoforms by expression of Cre recombinase in Stx1A/1B cDKO neurons essentially abolished EPSCs (Fig. 5B).
Among 27 Stx1A/1B cDKO neurons, 22 of them exhibited no evoked transmitter release and the remaining five had an average EPSC of $0.21 \pm 0.06 \mathrm{nA}$. Furthermore, measurements of the RRP of vesicles evoked by $5 \mathrm{~s}$ application of $500 \mathrm{~mm}$ hypertonic solution revealed a complete absence of fusion-competent vesicles in Stx1A/1B cDKO neurons expressing Cre recombinase, whereas the control groups exhibited RRPs of an average size of $\sim 0.4 \mathrm{nC}$ (Fig. 5C). Similarly, spontaneous release was also comparable among the control groups, as revealed by comparable mEPSC frequencies, whereas no mEPSC events were detected in Stx1A/1B cDKO neurons expressing Cre recombinase (Fig. 5D). These data indicate that the neuronal t-SNARE protein Stx1 is absolutely required for vesicle priming and fusion.

\section{Stx1's function in neuronal survival is independent of its function in neurotransmission}

Having completely eliminated Stx1 expression, we wanted to test whether the function of Stx 1 in neuronal survival and in synaptic transmission are coupled. Whereas the observation of the degeneration of synaptically silent mutant Munc18-1 KO neurons led to the hypothesis that synaptic activity is required for neuronal maintenance (Verhage et al., 2000), unimpaired survival of Munc13-1/2 DKO provided counterevidence for this hypothesis (Varoqueaux et al., 2002). Furthermore, a Stxl mutant with 2 point mutations interchanging the +4 and +5 hydrophobic layers of the SNARE motif (A240V, V244A; Stx1A ${ }^{\mathrm{AV}}$ ) is embryonically lethal in flies despite maintaining neurotransmitter release albeit at severely reduced levels (Fergestad et al., 2001). The degeneration phenotype of this mutant has been also shown by its inability to rescue survival of rat neurons subjected to botulinum 
A
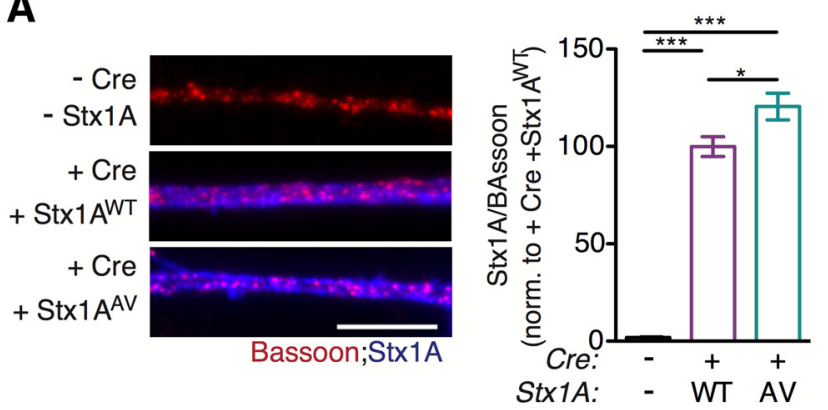

C
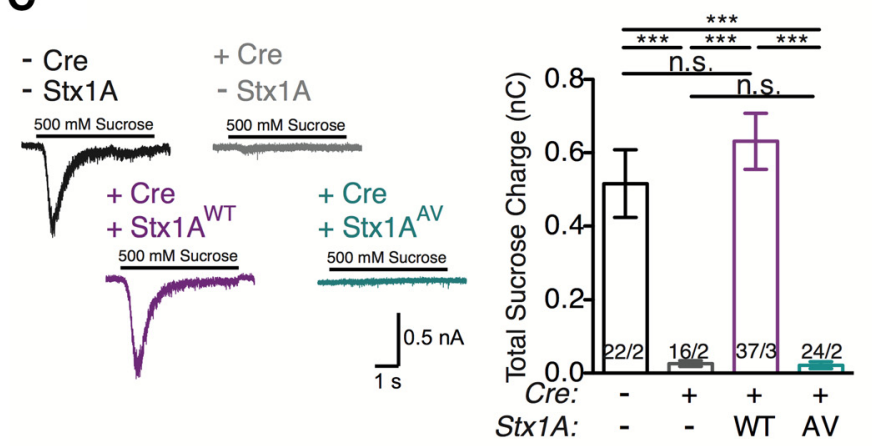

B

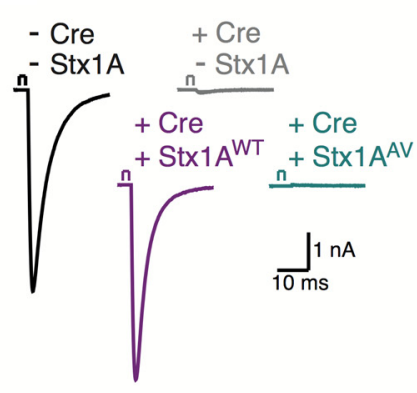

D

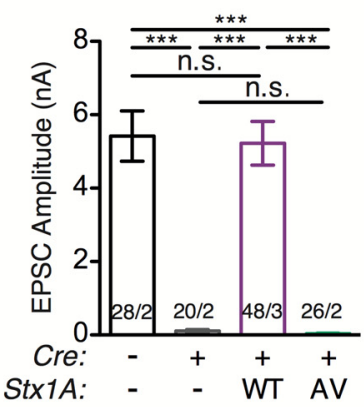

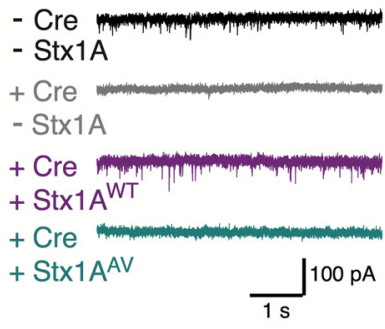

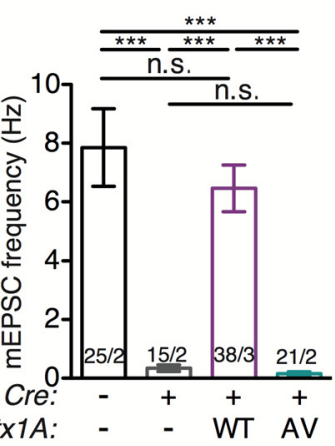

\section{$\mathbf{E}$}
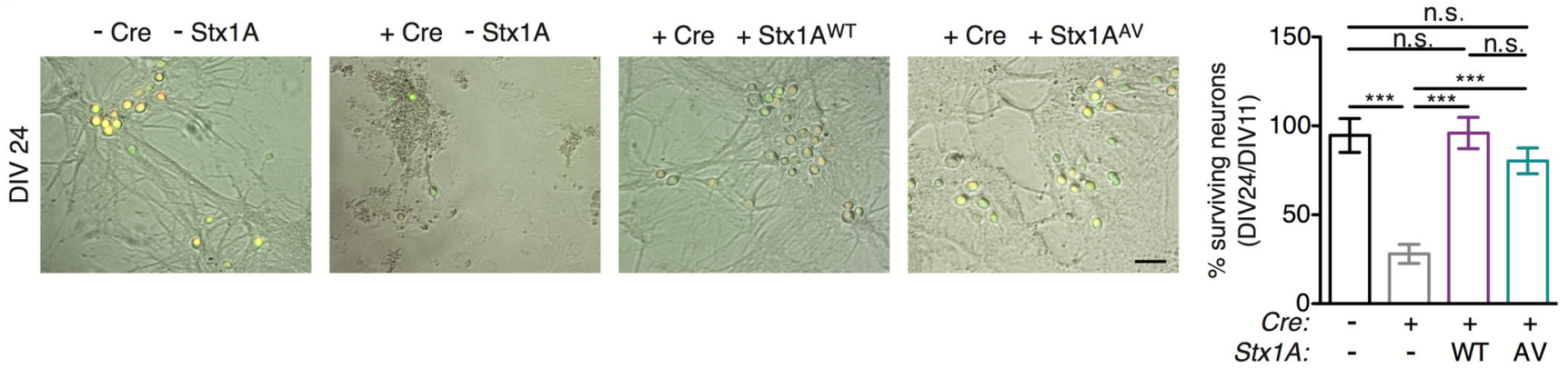

Figure 6. Stx 1 function in neuronal survival is independent from its function in neurotransmission. $A$, Example images (left) and summary graph (right) of immunofluorescence labeling for $S t x 1 A$ (blue) in Stx1A/1B cDK0 neurons coinfected at DIV 3 with viruses to express only the reporter constructs or Cre recombinase and Stx $1 A^{W T}$ or Stx $1 A^{A V}$ and fixed at DIV 14 . The average intensities of Stx1A immunofluorescence signals (blue) were measured at compartments positive for Bassoon immunofluorescence signal (red). $n=52-55$ images from three independent cultures. Scale bar, $10 \mu \mathrm{m} . \boldsymbol{B}$, Example traces (left) and summary graph (right) of EPSCS. C, Example traces (left) and summary graph (right) of RRPs. D, Example traces (left) and summary graph (right) of mEPSCs. $\boldsymbol{E}$, Example images of Stx 1A/1B cDKO cultures at DIV 24 (left) and summary graph of neuronal survival determined as the percentage of cell number at DIV 24/DIV11 (right). Scale bar, $50 \mu \mathrm{m} . n=60$ images from three independent cultures. Bar graphs show means \pm SEM; ${ }^{*} p \leq 0.05,{ }^{* * *} p \leq 0.001$. Cell numbers and the number of independent cultures are presented in the graphs in $\boldsymbol{B}-\boldsymbol{D}$. Artifacts and/or action potentials are blanked in $\boldsymbol{B}$ and $\boldsymbol{C}$. The traces were filtered at $1 \mathrm{kHz}$ in $\boldsymbol{D}$.

neurotoxin C (Peng et al., 2013). With the purpose of finding a Stx1 mutant, which maintains neurotransmitter release, albeit severely impaired, but which is noneffective for neuronal maintenance and thereby to split the functions of Stx1 in neurotransmitter release and neuronal maintenance, we coexpressed Stx1A ${ }^{\mathrm{AV}}$ with Cre recombinase in Stx1A/B cDKO neurons. For that purpose, we first examined the exogenous expression efficiencies of Stx $1 A^{\mathrm{AV}}$ and WT Stx1A (Stx1A $\left.{ }^{\mathrm{WT}}\right)$ in Stx1A/1B cDKO neurons expressing Cre recombinase. Immunofluorescence labeling of Stx1 $\mathrm{A}^{\mathrm{AV}}$ revealed an $\sim 20 \%$ higher signal on average compared with the Stx $1 A^{\text {WT }}$ at compartments positive for Bassoon signal, indicating that the exogenously expressed Stx $1 \mathrm{~A}^{\mathrm{AV}}$ can localize at synapses (Fig. 6A).

We next tested whether neurotransmitter release can be rescued by Stx $1 A^{A V}$ compared with Stx $1 A^{\mathrm{WT}}$. Expression of Stx $1 A^{\text {WT }}$ in Stx1A/1B cDKO neurons successfully restored evoked and spontaneous neurotransmitter release, as well as the RRP, back to the levels of control neurons, which includes only endogenous Stx1B, demonstrating again a functional redundancy between Stx1A and Stx1B in terms of neurotransmitter release (Fig. $6 B-D$ ). Conversely, Stx $1 A^{\mathrm{AV}}$ completely failed to rescue evoked and spontaneous release, nor could it restore the RRP in Stx1A/1B cDKO neurons (Fig. 6B-D). Given that Stx $1 \mathrm{~A}^{\mathrm{AV}}$ assembles into SNARE complexes, but with reduced stability (Kee et al., 1995; Fergestad et al., 2001), and that, in Stx1 AV , the mutated hydrophobic layers are closer to the $\mathrm{C}$ terminus, these data suggest that incomplete SNARE complex formation is not sufficient for vesicle fusion or for vesicle priming.

We therefore wanted to address whether Stx $1 \mathrm{~A}^{\mathrm{AV}}$ and, indirectly, whether partial SNARE complex assembly could rescue neuronal survival despite being release incompetent. We induced coexpression of Cre recombinase with $\operatorname{Stx} 1 \mathrm{~A}^{\mathrm{AV}}$ or with $\mathrm{Stx} 1 \mathrm{~A}^{\mathrm{WT}}$ by infecting Stx1A/1B cDKO neurons with viruses at DIV 1. The neurons were then counted at DIV 11 and DIV 24 and the ratio of 
A

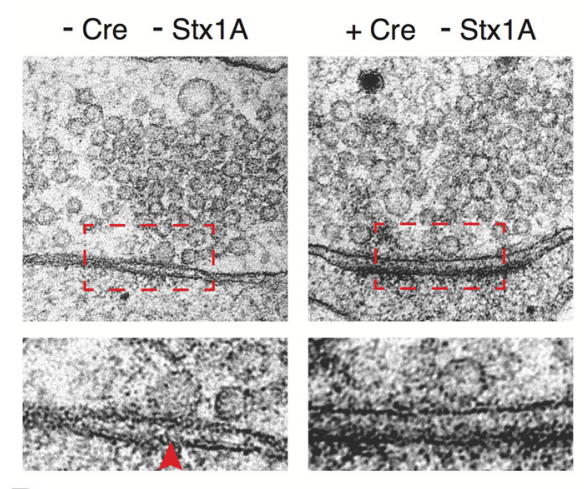

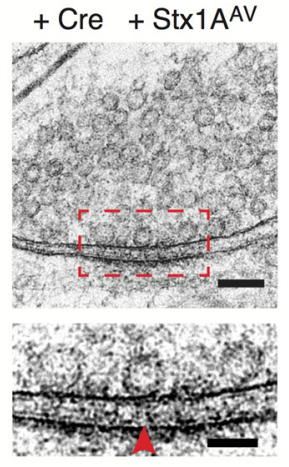

B

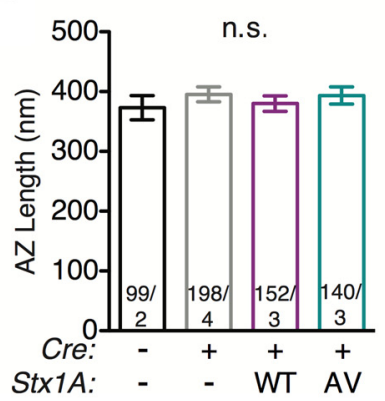

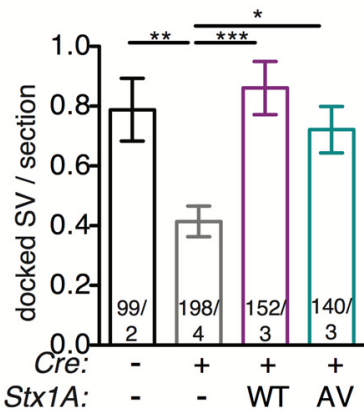

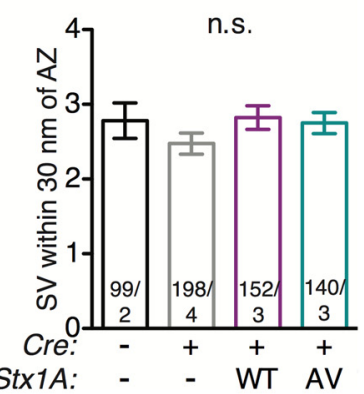

Figure 7. Stx1 functions in vesicle docking and fusion can be uncoupled. $A$, Example electron microscopy images of presynaptic nerve terminals from Stx1A/1B cDK0 neurons coinfected at DIV2 with viruses to express the reporter constructs, Cre recombinase and only a reporter construct, and Cre recombinase and Stx $1 A^{W T}$ or Stx $1 A^{A V}$. Arrows show the docked vesicles in the zoomed images. Scale bars, $100 \mathrm{~nm}$ (top) and $50 \mathrm{~nm}$ (bottom). B, Summary graphs of AZ length, docked synaptic vesicles (SVs) per section, and SVs within $30 \mathrm{~nm}$ of AZs. Bar graphs show means \pm SEM. Statistical analysis was applied by one-way ANOVA followed by Tukey's post hoc test; ${ }^{*} p \leq 0.05,{ }^{* *} p \leq 0.01,{ }^{* * *} p \leq 0.001$. The number of sections analyzed and the number of independent cultures are presented in the graphs.

the cell numbers at DIV 24/DIV 11 was used as a read-out for neuronal cell survival. Stx1A ${ }^{\mathrm{WT}}$ successfully rescued the Stx1A/1B cDKO neurons against cell death, showing again functional redundancy with Stx1B. Surprisingly, Stx $1 \mathrm{~A}^{\mathrm{AV}}$ also rescued neuronal survival at levels comparable to the control and Stx $1 A^{\mathrm{WT}}$ rescue (Fig. $6 E$ ). The inability of $\operatorname{Stx} 1 \mathrm{~A}^{\mathrm{AV}}$ to rescue botulinum neurotoxin-mediated neuronal death might be due to the additional mutation, which was included to render Stx1A cleavage resistant (Peng et al., 2013). Conversely, the efficiency of Stx1 $\mathrm{A}^{\mathrm{AV}}$ to rescue neuronal survival in our assay, but not neurotransmitter release, argues that the lack of synaptic activity is not a cause of neuronal degeneration and that Stx1 has independent functions in neuronal maintenance and neurotransmission. It may also suggest that partial SNARE complex assembly is sufficient for neuronal maintenance, but not for vesicle fusion and priming.

\section{Stx1 function in vesicle docking and fusion can be uncoupled} In addition to its functions in neuronal maintenance, vesicle fusion, and vesicle priming, Stxl plays a role in vesicle docking in mammalian neurons (Gerber et al., 2008; Arancillo et al., 2013; Imig et al., 2014). However, a reduction of more than $\sim 70 \%$ in Stx1 expression is required for observable impairments in vesicle docking (Arancillo et al., 2013; Imig et al., 2014), whereas unfeasibility of complete elimination of Stx1 has obscured the analysis of vesicle docking in the absence of Stx1. To evaluate the impact of complete loss of Stx1 in vesicle docking, we performed highpressure freezing experiments of the cultured Stx1A/1B cDKO hippocampal neurons infected with viruses at DIV 2 and fixed at
DIV 12-14. We then examined the ultrastructure of the synapses by using electron microscopy. Stx1A/1B cDKO neurons expressing Cre recombinase revealed a $\sim 50 \%$ reduction in the number of docked vesicles compared with the control neurons, whereas no significant differences were observed in AZ length and total vesicle number within $30 \mathrm{~nm} \mathrm{AZ} \mathrm{(Fig.} \mathrm{7).}$ Even though our results point toward a further reduction in vesicle docking compared with previous studies, which have analyzed the effects of severely reduced levels of Stx1B on Stx1A-null background in mice (Arancillo et al., 2013; Imig et al., 2014), the impairment in vesicle docking is not as severe as observed in Munc13-1/2 DKO neurons (Imig et al., 2014). However, our analysis in a conditional KO system is hampered by cytotoxic effects of loss of Stx1 and thus biased toward cells resembling severe hypomorphic expression of Stx1. Although it is evident that Stx1 is essential for vesicle docking, we cannot rule out the involvement of residual Stx1B in the manifestation of a remaining vesicle-docking function.

Although our data demonstrate that full SNARE complex assembly is absolutely essential for vesicle fusion and vesicle priming, as manifested by complete inhibition of neurotransmission by Stx $1 A^{A V}$ (Fig. 6), the role of SNARE complex assembly in vesicle docking is still not well defined. To test whether vesicle docking can be recovered in Stx1A/1B cDKO neurons by Stx $1 \mathrm{~A}^{\mathrm{AV}}$ and thus by putative partial SNARE complex assembly, we coexpressed Stx $1 A^{\text {WT }}$ or Stx $1 A^{A V}$ with Cre recombinase. Expression of Stx $1 A^{\text {WT }}$ fully restored vesicle docking back to the control levels, providing evidence for functional redundancy between Stx1A and Stx1B also in terms of vesicle docking (Fig. 7). Remarkably, expression of Stx1A ${ }^{\mathrm{AV}}$ completely restored docked vesicles to levels comparable to the control neurons, demonstrating a clear functional difference between vesicle docking and priming. More importantly, the ability of Stx $1 \mathrm{~A}^{\mathrm{AV}}$ to dock the vesicles successfully to the plasma membrane is suggestive of the sufficiency of partially assembled SNARE complexes for vesicle docking.

\section{Discussion}

The fundamental roles of Stx 1 in neuronal maintenance and neurotransmission have been well established by genetic manipulation studies in invertebrates (Schulze et al., 1995; Burgess et al., 1997; Saifee et al., 1998). The situation in mammalian neurons is less clear. The redundancy between the two isoforms Stx1A and Stx1B (Fujiwara et al., 2006; Gerber et al., 2008; Arancillo et al., 2013; Zhou et al., 2013; Wu et al., 2015) and the embryonic lethality in Stx1A/1B DKO mice (Mishima et al., 2014) limited the functional studies of Stx1 only to the conditions of severely reduced expression of Stx1 (Arancillo et al., 2013; Zhou et al., 2013). With our Stx1A/1B DKO mouse models, we bypassed these limitations and investigated the impacts of loss of Stx1 on neurotransmission and neuronal maintenance in a mammalian system. We draw several major conclusions. Stx1 has an abso- 
lutely essential function in neuronal maintenance in vivo and in vitro, not only during development, but throughout the neuronal life span. This function is independent of its function in neurotransmission and the particular role of Stx1 in neurotransmission can be broken down into its functions in vesicle docking and vesicle fusion.

\section{Stx1 is absolutely required for neuronal maintenance}

Complete loss of Stx1 by deletion of both Stx1A and Stx1B led to embryonic lethality, which is consistent with a recent study (Mishima et al., 2014) and caused widespread cell loss in embryonic mouse brains as well as premature neuronal lethality in vitro (Figs. 1, 2). In addition, in vitro genetic removal of Stx1 subsequently caused death of differentiated neurons, demonstrating the importance of Stx 1 also for the neuronal maintenance after maturation.

An equal severity of neuronal lethality was not observed so far in other vesicular SNARE KO mutants because loss of Syb2 or SNAP-25 shows no signs of neuronal loss in vivo (Schoch et al., 2001; Washbourne et al., 2002), with the latter leading to neuronal death only in vitro (Delgado-Martínez et al., 2007). Conversely, loss of Munc18-1, a well defined interaction partner of Stx1, produces a phenocopy of loss of Stx 1 with severe neuronal lethality (Verhage et al., 2000), suggesting a functional cooperation between Stx1 and Munc18-1 in neuronal maintenance.

What is the function of Stxl in neuronal maintenance and is it coupled to its function in neurotransmitter release? Previous studies suggest a primary dysfunction in trophic support to be the underlying mechanism of Stx1-mediated cell death (Kofuji et al., 2014); however, a putative glial dysfunction is incompatible with our experiments because we cocultured the Stx1-deficient neurons with WT astrocytes. Furthermore, selective death of Munc18-1-deficient Purkinje cells in vivo showed the cellautonomous nature of the observed cell loss (Heeroma et al., 2004). Nevertheless, we cannot rule out the involvement of dysfunctions in autocrine signaling pathways.

Lack of synaptic input has also been proposed as a possible mechanism leading to neuronal death (Verhage et al., 2000), yet the unimpaired viability of Syb1/2-deficient and Munc13-1/-2deficient neurons, despite being release incompetent (Varoqueaux et al., 2002; Zimmermann et al., 2014), shows that neuronal maintenance is independent of neurotransmission. Interestingly, in our conditional Stx 1 deletion model, neuronal survival was rescued not only by WT Stx 1, but also by a Stx1 mutant (A240V, V244A; Stx1A ${ }^{\mathrm{AV}}$ ) that is fully deficient in neurotransmitter release (Fig. 6). In view of these findings, we suggest that Stx1, in conjunction with Munc18-1, is required for survival, but in a function that is not related to neurotransmitter release, such as in the ER, Golgi, and posttranslational trafficking processes (Rowe et al., 1999, 2001; Toonen et al., 2005; Arunachalam et al., 2008), in nonvesicular lipid trafficking, and/or in the ER positioning at the plasma membrane contact sites (Petkovic et al., 2014). Defects in these systems would in turn lead to neuronal death. Moreover, Stx1 interacts with glycine and GABA transporters (Blakely et al., 1998; López-Corcuera et al., 2001) and modulates N- and P/Q-type $\mathrm{Ca}^{2+}$-channels (Lévêque et al., 1994; Sheng et al., 1994) and M-type $\mathrm{K}^{+}$-channels (Regev et al., 2009; Etzioni et al., 2011). A shift in the ionic homeostasis, such as excess amounts of intracellular $\mathrm{Ca}^{2+}$, could also produce cytotoxic effects (Valentino et al., 1993; Cataldi, 2013).

A direct cooperation of Stx 1 and Munc18-1 in neuronal maintenance is not proven, but likely. As costabilizing chaperones
(Rowe et al., 1999, 2001; Toonen et al., 2005), the putative cooperation of Munc18-1 and Stx1 may in principle be based on an upstream-downstream relationship as implied by our crossrescue experiments (Fig. 3). Differential efficiencies of Stx1A and Stx1B to rescue Munc18-1 KO neurons (Fig. 3D,E) also points toward a correlation between Munc18-1-dependent Stx1 isoform stability and the severity of phenotype. In fact, loss of Stx1B isoforms is more deleterious than loss of Stx1A (Kofuji et al., 2014; Mishima et al., 2014; Wu et al., 2015) and a decrease in Munc18-1 protein levels is observed only as a result of loss or severe reduction of Stx1B (Gerber et al., 2008; Arancillo et al., 2013; Wu et al., 2015), not as a result of loss of Stx1A (Fujiwara et al., 2006; Gerber et al., 2008). However, the ultimate death of either Stx1- or Munc18-1 deficient neurons is incompatible with such an upstream-downstream relationship. Alternatively, a partial redundancy of Munc18-1 with another isoform, such as Munc18-2, could explain the differential cross rescue activities of Stx1 and Munc18-1. Even though Munc18-2 is not expressed at detectable levels in neurons (Hata and Südhof, 1995), it has been shown to be able to bind to Stx1 (Hata and Südhof, 1995; Rowe et al., 2001; Gulyás-Kovács et al., 2007) and to be expressed at elevated levels in Munc18-1 knock-down PC12 cells (Arunachalam et al., 2008), yet the expression pattern of Munc18 isoforms in the developing brain remains to be elucidated.

\section{Differential functions of Stx1 in vesicle docking and fusion}

With our Stx1A/1B cDKO mouse model, we identified a time window when endogenous Stxl was completely lost with no apparent signs of degenerated neuronal structures, providing the suitable conditions for studies of Stx1's functions in neurotransmission. Electrophysiological recordings from Stx1A/1B cDKO hippocampal neurons revealed complete elimination of neurotransmitter release, including spontaneous and synchronous or asynchronous evoked release (Fig. 5). In addition, vesicle priming was completely inhibited as shown by lack of fusion competent vesicles (Fig. 5). However, a recent study with a similar Stx1A/1B DKO mouse model showed residual asynchronous synaptic activity (Mishima et al., 2014), supporting a model in which release can occur in the absence of Stx 1 . The asynchronous release observed by Mishima et al. (2014) might be due to upregulation of, or compensation by, another Stx isoform in a subpopulation of embryonic cortical neurons. Indeed, similar issues of redundancies have also been observed for the other two mammalian synaptic SNAREs. Remaining release activity in SNAP25-deficient neurons (Washbourne et al., 2002) may well be mediated by SNAP-23 (Delgado-Martínez et al., 2007). In addition, Syb2 is partially redundant to Syb1 and the latter is responsible for remaining release activity (Schoch et al., 2001; Zimmermann et al., 2014) and vesicle-docking activity (Imig et al., 2014) in Syb2 KO neurons. Together, our results fully support the canonical role of SNAREs for any form of neurotransmission.

We also used the Stx1A/1B cDKO models for functional reconstitution experiments. Although exogenous expression of WT Stx1A completely restored vesicle fusion and priming in Stx1A/1B cDKO neurons, the Stx1 ${ }^{\mathrm{AV}}$ mutant failed to do so. By the position of the mutations A240V, V244A, which lay at the +4 and +5 layers of SNARE motif, it is plausible that Stx $1 \mathrm{~A}^{\mathrm{AV}}$ would assemble with the other SNARE proteins into SNARE complexes. In fact, it has been demonstrated by in vitro experiments that Stx1A ${ }^{\text {AV }}$ forms ternary SNARE complexes with SNAP-25 and Syb-2 (Kee et al., 1995; Fergestad et al., 2001). However, the reduced stability of the SNARE complexes containing Stx1A ${ }^{\mathrm{AV}}$ (Kee et al., 1995; Fergestad et al., 2001) would suggest impair- 
ment in full assembly, which in turn may explain the inhibition of neurotransmitter release in our study.

Remarkably, Stx $1 \mathrm{~A}^{\mathrm{AV}}$ was able to fully rescue vesicle docking to WT levels in Stx1A/1B cDKO neurons, yet the vesicles were fusion incompetent. This differential effect strongly supports two hypotheses: (1) vesicle docking and vesicle priming are two distinct functional steps that can be differentially assessed by morphological and electrophysiological analysis and (2) vesicle docking is less affected by the stability of SNARE complexes than neurotransmitter release. These phenomena can be explained by the sequential zippering mechanism: SNARE complex assembly is mediated by zippering from the $\mathrm{N}$ to the $\mathrm{C}$ terminus and partially assembled SNARE complexes are arrested before $\mathrm{Ca}^{2+}$ entry. In fact, structural analyses using nuclear magnetic resonance or atomic force microscopies depicted a sequential SNARE complex formation proceeding in two or three steps (Ellena et al., 2009; Gao et al., 2012; Liang et al., 2013). In addition, mutational analysis of SNAP-25 and Syb2 in neurons and chromaffin cells revealed the critical role of the layers from +4 to +6 of the SNARE complex in neurotransmitter release, supporting the hypothesis of sequential assembly of the SNARE complex (Sørensen et al., 2006; Walter et al., 2010). Consistent with these observations, we show here that docking and priming/fusion are associated with different SNARE assembly states, in which the docking state does not require an intact layer $+4 /+5$ structure. Overall, this suggests that these residues might reside between two separate functional domains in the SNARE complex, which are separately responsible for vesicle docking and priming.

\section{References}

Arancillo M, Min SW, Gerber S, Münster-Wandowski A, Wu YJ, Herman M, Trimbuch T, Rah JC, Ahnert-Hilger G, Riedel D, Südhof TC, Rosenmund C (2013) Titration of Syntaxin1 in mammalian synapses reveals multiple roles in vesicle docking, priming, and release probability. J Neurosci 33:16698-16714. CrossRef Medline

Arunachalam L, Han L, Tassew NG, He Y, Wang L, Xie L, Fujita Y, Kwan E, Davletov B, Monnier PP, Gaisano HY, Sugita S (2008) Munc18-1 is critical for plasma membrane localization of syntaxin 1 but not of SNAP-25 in PC12 cells. Mol Biol Cell 19:722-734. Medline

Bennett MK, Calakos N, Scheller RH (1992) Syntaxin: a synaptic protein implicated in docking of synaptic vesicles at presynaptic active zones. Science 257:255-259. CrossRef Medline

Blakely RD, Ramamoorthy S, Schroeter S, Qian Y, Apparsundaram S, Galli A, DeFelice LJ (1998) Regulated phosphorylation and trafficking of antidepressant-sensitive serotonin transporter proteins. Biol Psychiatry 44:169-178. CrossRef Medline

Burgess RW, Deitcher DL, Schwarz TL (1997) The synaptic protein syntaxin1 is required for cellularization of Drosophila embryos. J Cell Biol 138:861-875. CrossRef Medline

Cataldi M (2013) The changing landscape of voltage-gated calcium channels in neurovascular disorders and in neurodegenerative diseases. Curr Neuropharmacol 11:276-297. CrossRef Medline

Delgado-Martínez I, Nehring RB, Sørensen JB (2007) Differential abilities of SNAP-25 homologs to support neuronal function. J Neurosci 27:93809391. CrossRef Medline

Ellena JF, Liang B, Wiktor M, Stein A, Cafiso DS, Jahn R, Tamm LK (2009) Dynamic structure of lipid-bound synaptobrevin suggests a nucleationpropagation mechanism for trans-SNARE complex formation. Proc Natl Acad Sci U S A 106:20306-20311. CrossRef Medline

Etzioni A, Siloni S, Chikvashvilli D, Strulovich R, Sachyani D, Regev N, Greitzer-Antes D, Hirsch JA, Lotan I (2011) Regulation of neuronal $\mathrm{M}$-channel gating in an isoform-specific manner: functional interplay between calmodulin and syntaxin 1A. J Neurosci 31:14158-14171. CrossRef Medline

Fergestad T, Wu MN, Schulze KL, Lloyd TE, Bellen HJ, Broadie K (2001) Targeted mutations in the syntaxin $\mathrm{H} 3$ domain specifically disrupt SNARE complex function in synaptic transmission. J Neurosci 21:91429150. Medline
Fujiwara T, Mishima T, Kofuji T, Chiba T, Tanaka K, Yamamoto A, Akagawa K (2006) Analysis of knock-out mice to determine the role of HPC-1/ syntaxin 1A in expressing synaptic plasticity. J Neurosci 26:5767-5776. CrossRef Medline

Gao Y, Zorman S, Gundersen G, Xi Z, Ma L, Sirinakis G, Rothman JE, Zhang Y (2012) Single reconstituted neuronal SNARE complexes zipper in three distinct stages. Science 337:1340-1343. CrossRef Medline

Gerber SH, Rah JC, Min SW, Liu X, de Wit H, Dulubova I, Meyer AC, Rizo J, Arancillo M, Hammer RE, Verhage M, Rosenmund C, Südhof TC (2008) Conformational switch of syntaxin- 1 controls synaptic vesicle fusion. Science 321:1507-1510. CrossRef Medline

Gulyás-Kovács A, de Wit H, Milosevic I, Kochubey O, Toonen R, Klingauf J, Verhage M, Sørensen JB (2007) Munc18-1: sequential interactions with the fusion machinery stimulate vesicle docking and priming. J Neurosci 27:8676-8686. CrossRef Medline

Harrison SD, Broadie K, van de Goor J, Rubin GM (1994) Mutations in the Drosophila Rop gene suggest a function in general secretion and synaptic transmission. Neuron 13:555-566. CrossRef Medline

Hata Y, Südhof TC (1995) A novel ubiquitous form of Munc-18 interacts with multiple syntaxins. Use of the yeast two-hybrid system to study interactions between proteins involved in membrane traffic. J Biol Chem 270:13022-13028. CrossRef Medline

Hata Y, Slaughter CA, Südhof TC (1993) Synaptic vesicle fusion complex contains unc-18 homologue bound to syntaxin. Nature 366:347-351. CrossRef Medline

Heeroma JH, Roelandse M, Wierda K, van Aerde KI, Toonen RF, Hensbroek RA, Brussaard A, Matus A, Verhage M (2004) Trophic support delays but does not prevent cell-intrinsic degeneration of neurons deficient for Munc18-1. Eur J Neurosci 20:623-634. CrossRef Medline

Imig C, Min SW, Krinner S, Arancillo M, Rosenmund C, Südhof TC, Rhee J, Brose N, Cooper BH (2014) The morphological and molecular nature of synaptic vesicle priming at presynaptic active zones. Neuron 84:416-431. CrossRef Medline

Kee Y, Lin RC, Hsu SC, Scheller RH (1995) Distinct domains of syntaxin are required for synaptic vesicle fusion complex formation and dissociation. Neuron 14:991-998. CrossRef Medline

Kim JH, Lee SR, Li LH, Park HJ, Park JH, Lee KY, Kim MK, Shin BA, Choi SY (2011) High cleavage efficiency of a 2A peptide derived from porcine teschovirus-1 in human cell lines, zebrafish and mice. PLoS One 6:e18556. CrossRef Medline

Kofuji T, Fujiwara T, Sanada M, Mishima T, Akagawa K (2014) HPC-1/ syntaxin $1 \mathrm{~A}$ and syntaxin $1 \mathrm{~B}$ play distinct roles in neuronal survival. J Neurochem 130:514-525. CrossRef Medline

Lévêque C, el Far O, Martin-Moutot N, Sato K, Kato R, Takahashi M, Seagar MJ (1994) Purification of the N-type calcium channel associated with syntaxin and synaptotagmin. A complex implicated in synaptic vesicle exocytosis. J Biol Chem 269:6306-6312. Medline

Liang B, Kiessling V, Tamm LK (2013) Prefusion structure of syntaxin-1A suggests pathway for folding into neuronal trans-SNARE complex fusion intermediate. Proc Natl Acad Sci U S A 110:19384-19389. CrossRef Medline

Lois C, Hong EJ, Pease S, Brown EJ, Baltimore D (2002) Germline transmission and tissue-specific expression of transgenes delivered by lentiviral vectors. Science 295:868-872. CrossRef Medline

López-Corcuera B, Aragón C, Geerlings A (2001) Regulation of glycine transporters. Biochem Soc Trans 29:742-745. CrossRef Medline

Mishima T, Fujiwara T, Sanada M, Kofuji T, Kanai-Azuma M, Akagawa K (2014) Syntaxin 1B, but not syntaxin 1A, is necessary for the regulation of synaptic vesicle exocytosis and of the readily releasable pool at central synapses. PLoS One 9:e90004. CrossRef Medline

Peng L, Liu H, Ruan H, Tepp WH, Stoothoff WH, Brown RH, Johnson EA, Yao WD, Zhang SC, Dong M (2013) Cytotoxicity of botulinum neurotoxins reveals a direct role of syntaxin 1 and SNAP-25 in neuron survival. Nat Commun 4:1472. CrossRef Medline

Petkovic M, Jemaiel A, Daste F, Specht CG, Izeddin I, Vorkel D, Verbavatz JM, Darzacq X, Triller A, Pfenninger KH, Tareste D, Jackson CL, Galli T (2014) The SNARE Sec22b has a non-fusogenic function in plasma membrane expansion. Nat Cell Biol 16:434-444. CrossRef Medline

Regev N, Degani-Katzav N, Korngreen A, Etzioni A, Siloni S, Alaimo A, Chikvashvili D, Villarroel A, Attali B, Lotan I (2009) Selective interaction of syntaxin 1A with KCNQ2: possible implications for specific modulation of presynaptic activity. PLoS One 4:e6586. CrossRef Medline 
Rizo J, Rosenmund C (2008) Synaptic vesicle fusion. Nat Struct Mol Biol 15:665-674. CrossRef Medline

Rizo J, Südhof TC (2012) The membrane fusion enigma: SNAREs, Sec1/ Munc18 proteins, and their accomplices-guilty as charged? Annu Rev Cell Dev Biol 28:279-308. CrossRef Medline

Rosenmund C, Stevens CF (1996) Definition of the readily releasable pool of vesicles at hippocampal synapses. Neuron 16:1197-1207. CrossRef Medline

Rowe J, Corradi N, Malosio ML, Taverna E, Halban P, Meldolesi J, Rosa P (1999) Blockade of membrane transport and disassembly of the Golgi complex by expression of syntaxin $1 \mathrm{~A}$ in neurosecretion-incompetent cells: prevention by rbSEC1. J Cell Sci 112:1865-1877. Medline

Rowe J, Calegari F, Taverna E, Longhi R, Rosa P (2001) Syntaxin 1A is delivered to the apical and basolateral domains of epithelial cells: the role of munc-18 proteins. J Cell Sci 114:3323-3332. Medline

Saifee O, Wei L, Nonet ML (1998) The Caenorhabditis elegans unc-64 locus encodes a syntaxin that interacts genetically with synaptobrevin. Mol Biol Cell 9:1235-1252. CrossRef Medline

Schoch S, Deák F, Königstorfer A, Mozhayeva M, Sara Y, Südhof TC, Kavalali ET (2001) SNARE function analyzed in synaptobrevin/VAMP knockout mice. Science 294:1117-1122. CrossRef Medline

Schulze KL, Bellen HJ (1996) Drosophila syntaxin is required for cell viability and may function in membrane formation and stabilization. Genetics 144:1713-1724. Medline

Schulze KL, Broadie K, Perin MS, Bellen HJ (1995) Genetic and electrophysiological studies of Drosophila syntaxin-1A demonstrate its role in nonneuronal secretion and neurotransmission. Cell 80:311-320. CrossRef Medline

Sheng ZH, Rettig J, Takahashi M, Catterall WA (1994) Identification of a syntaxin-binding site on N-type calcium channels. Neuron 13:13031313. CrossRef Medline

Sørensen JB, Wiederhold K, Müller EM, Milosevic I, Nagy G, de Groot BL, Grubmüller H, Fasshauer D (2006) Sequential N- to C-terminal SNARE complex assembly drives priming and fusion of secretory vesicles. EMBO J 25:955-966. CrossRef Medline

Toonen RF, de Vries KJ, Zalm R, Südhof TC, Verhage M (2005) Munc18-1 stabilizes syntaxin 1 , but is not essential for syntaxin 1 targeting and SNARE complex formation. J Neurochem 93:1393-1400. CrossRef Medline

Valentino K, Newcomb R, Gadbois T, Singh T, Bowersox S, Bitner S, Justice
A, Yamashiro D, Hoffman BB, Ciaranello R (1993) A selective N-type calcium channel antagonist protects against neuronal loss after global cerebral ischemia. Proc Natl Acad Sci U S A 90:7894-7897. CrossRef Medline

Varoqueaux F, Sigler A, Rhee JS, Brose N, Enk C, Reim K, Rosenmund C (2002) Total arrest of spontaneous and evoked synaptic transmission but normal synaptogenesis in the absence of Munc13-mediated vesicle priming. Proc Natl Acad Sci U S A 99:9037-9042. CrossRef Medline

Varoqueaux F, Sons MS, Plomp JJ, Brose N (2005) Aberrant morphology and residual transmitter release at the Munc13-deficient mouse neuromuscular synapse. Mol Cell Biol 25:5973-5984. CrossRef Medline

Verhage M, Maia AS, Plomp JJ, Brussaard AB, Heeroma JH, Vermeer H, Toonen RF, Hammer RE, van den Berg TK, Missler M, Geuze HJ, Südhof TC (2000) Synaptic assembly of the brain in the absence of neurotransmitter secretion. Science 287:864-869. CrossRef Medline

Walter AM, Wiederhold K, Bruns D, Fasshauer D, Sørensen JB (2010) Synaptobrevin N-terminally bound to syntaxin-SNAP-25 defines the primed vesicle state in regulated exocytosis. J Cell Biol 188:401-413. CrossRef Medline

Washbourne P, Thompson PM, Carta M, Costa ET, Mathews JR, LópezBenditó G, Molnár Z, Becher MW, Valenzuela CF, Partridge LD, Wilson MC (2002) Genetic ablation of the t-SNARE SNAP-25 distinguishes mechanisms of neuroexocytosis. Nat Neurosci 5:19-26. Medline

Watanabe S, Liu Q, Davis MW, Hollopeter G, Thomas N, Jorgensen NB, Jorgensen EM (2013) Ultrafast endocytosis at Caenorhabditis elegans neuromuscular junctions. Elife 2:e00723. CrossRef Medline

Wu YJ, Tejero R, Arancillo M, Vardar G, Korotkova T, Kintscher M, Schmitz D, Ponomarenko A, Tabares L, Rosenmund C (2015) Syntaxin 1B is important for mouse postnatal survival and proper synaptic function at the mouse neuromuscular junctions. J Neurophysiol 114:2404-2417. CrossRef Medline

Xue M, Reim K, Chen X, Chao HT, Deng H, Rizo J, Brose N, Rosenmund C (2007) Distinct domains of complexin I differentially regulate neurotransmitter release. Nat Struct Mol Biol 14:949-958. CrossRef Medline

Zhou P, Pang ZP, Yang X, Zhang Y, Rosenmund C, Bacaj T, Südhof TC (2013) Syntaxin-1 N-peptide and Habc-domain perform distinct essential functions in synaptic vesicle fusion. EMBO J 32:159-171. Medline

Zimmermann J, Trimbuch T, Rosenmund C (2014) Synaptobrevin 1 mediates vesicle priming and evoked release in a subpopulation of hippocampal neurons. J Neurophysiol 112:1559-1565. CrossRef Medline 